\title{
Twisted trace Paley-Wiener theorems for special and general linear groups
}

\author{
Paul Mezo
}

\begin{abstract}
Let $G$ be a real special or general linear group and $\sigma_{0}$ be the transpose-inverse involution. We characterize the image of $f \mapsto \operatorname{tr}\left(\pi(f) \pi\left(\sigma_{0}\right)\right)$ for irreducible representations $\pi$ of $G \rtimes\left\langle\sigma_{0}\right\rangle$, and $K$-finite $f \in C_{c}^{\infty}(G)$.
\end{abstract}

\section{Introduction}

From the perspective of representation theory, the Fourier transform of an integrable function $f$ is given by integration against the irreducible unitary representations $\pi$ of the real line

$$
\pi \mapsto \int_{\mathbb{R}} \pi(x) f(x) d x
$$

The classical Paley-Wiener theorem is a characterization of the image under the Fourier transform of the space of smooth compactly supported functions.

If, in place of the real line, one considers a connected real reductive group $G$, then the 'Fourier transform' of a smooth compactly supported function $f$ can be taken to be integration against the unitary representations $\pi$ induced from the discrete series or limits of discrete series. The resulting integral is an operator rather than a scalar, but this operator is of trace class so one can consider the transform

$$
\pi \mapsto \operatorname{tr}\left(\int_{G} \pi(x) f(x) d x\right)=\operatorname{tr}(\pi(f)) .
$$

Clozel and Delorme have characterized the image of this transform for smooth compactly supported functions which are finite under the action of a maximal compact subgroup [CD84, CD90]. This constitutes what is known as a trace Paley-Wiener theorem for $G$.

One can continue to generalize by supposing that $\sigma$ is an automorphism of $G$ of finite order. One can then consider the representations $\pi$ induced from the discrete series or limits of discrete series of the group $G \rtimes\langle\sigma\rangle$, and the transform

$$
\pi \mapsto \operatorname{tr}\left(\int_{G} \pi(x) f(x) d x \pi(\sigma)\right)=\operatorname{tr}(\pi(f) \pi(\sigma)) .
$$

A twisted trace Paley-Wiener theorem for $G$ is the characterization of the image of such a transform on the space of smooth compactly supported functions which are finite under the action of a maximal compact subgroup. Delorme has proven a twisted trace Paley-Wiener theorem when $G$ is a complex Lie group and $\sigma$ is the automorphism provided by complex conjugation. The goal of this work is to prove twisted trace Paley-Wiener theorems for $G$ equal to one of $\operatorname{SL}(n, \mathbb{R}), \mathrm{SL}^{ \pm}(n, \mathbb{R})$ or $\mathrm{GL}(n, \mathbb{R})$.

The principal result is a $\sigma_{0}$-twisted trace Paley-Wiener theorem (Theorem 1 ) in which $\sigma_{0}$ is the automorphism of $\operatorname{SL}(n, \mathbb{R})$ given by transposing and inverting. Instead of dealing with the group

Received 6 November 2001, accepted in final form 6 January 2003.

2000 Mathematics Subject Classification 22E30, $22 \mathrm{E} 46$.

Keywords: Paley-Wiener theorem, twisted, disconnected real groups.

This journal is (C) Foundation Compositio Mathematica 2004. 
$\mathrm{SL}(n, \mathbb{R}) \rtimes\left\langle\sigma_{0}\right\rangle$ and its representations, we work with $\operatorname{SL}(n, \mathbb{R})$ and its $\sigma_{0}$-stable representations. The two classes of representations are essentially the same (cf. [Del91, § 1]). Sections 3-5 are concerned with the identification of the $\sigma_{0}$-stable representations and $\S 6$ handles their associated intertwining operators. There are two families of intertwining operators, each of which is distinguished by a Weyl group which either includes or ignores an action of $\mathrm{R}$ groups. These two families lead to a dichotomy in the $\sigma_{0}$-twisted trace Paley-Wiener theorems of $\S 7$. In $\S 8$ we set forth a necessary compatibility condition and a conjecture, which together eliminate this dichotomy.

The final section is concerned with applications of the $\sigma_{0}$-twisted trace Paley-Wiener theorems. We begin by indicating how to obtain further twisted trace Paley-Wiener theorems for the groups $\mathrm{SL}(n, \mathbb{R})$ and $\mathrm{GL}(n, \mathbb{R})$. We conclude with an application needed for the twisted invariant ArthurSelberg trace formula. This application depends on the conjecture of $\S 8$ if the underlying group is $\mathrm{SL}(n, \mathbb{R})$ and $n$ is even. In all other cases, the application follows unconditionally.

\section{Preliminaries}

The reader is assumed to be familiar with the basic theory of Lie groups and their representations. We shall therefore set up our notation without references.

Set $G=\operatorname{SL}(n, \mathbb{R})$. The subgroup of upper-triangular matrices $P_{0}$ is a minimal parabolic subgroup of $G$. A parabolic subgroup $P \subset G$ is said to be standard if $P \supset P_{0}$. Suppose $P$ is a standard parabolic subgroup. Then it has a Levi decomposition $P=M U_{M}$, where $U_{M}$ is its unipotent radical and $M$ is a Levi subgroup containing $M_{0}$, the diagonal subgroup. The subgroup $P$ also has a Langlands decomposition $P=M^{1} A_{M} U_{M}$, in which $A_{M}$ is the connected component of the centre of $M$ and $M^{1}$ is the subgroup formed by the elements of $M$ having determinant \pm 1 . The subgroup $M^{1}$ is isomorphic to

$$
\left\{\left(x_{1}, \ldots, x_{\ell}\right) \in \mathrm{SL}^{ \pm}\left(n_{1}, \mathbb{R}\right) \times \cdots \times \mathrm{SL}^{ \pm}\left(n_{\ell}, \mathbb{R}\right): \prod_{j=1}^{\ell} \operatorname{det}\left(x_{j}\right)=1\right\},
$$

for some positive integers $n_{1}, \ldots, n_{\ell}$ such that $\sum_{j=1}^{\ell} n_{j}=n$. In other words, $M^{1}$ (and $M$ ) are block diagonal subgroups of $G$. We shall refer to the integers $n_{1}, \ldots, n_{\ell}$ as the block sizes of $M^{1}$ or $M$. We shall say that an element $x \in G$ permutes the blocks of $M$ if $x^{-1} M x$ is a Levi subgroup containing $M_{0}$, and has the same block sizes as $M$.

These notions make sense for arbitrary parabolic subgroups, but we shall only require them for standard parabolic subgroups. With this in mind, for any Levi subgroup $L$ containing $M_{0}$ define $P_{L}$ to be the unique standard parabolic subgroup whose Langlands decomposition is $P_{L}=L^{1} A_{L} U_{L}$. In particular, for $P$ and $M$ as above we have $P=P_{M}$.

We denote the Lie algebra of $A_{M}$ by $\mathfrak{a}_{M}$. Its dual is denoted by $\mathfrak{a}_{M}^{*}$. The complexification of a vector space shall be denoted by a subscript $\mathbb{C}$. For example, the complex dual of $\mathfrak{a}_{M}$ is $\mathfrak{a}_{M, \mathbb{C}}^{*}$. There is a canonical embedding of $\mathfrak{a}_{M}$ into $\mathfrak{a}_{M_{0}}$. The Killing form provides an inner product on $\mathfrak{a}_{M_{0}}$ which by restriction is an inner product on $\mathfrak{a}_{M}$. Given two Levi subgroups $L \subset M$ we write $\mathfrak{a}_{L}^{M}$ for the orthogonal dual of $\mathfrak{a}_{M}$ in $\mathfrak{a}_{L}$. We extend this notation to the duals and complex duals in the obvious way.

The set of (equivalence classes of) discrete series or non-degenerate limit of discrete series representations of $M^{1}$ is denoted by $(\hat{M})_{\text {lds }}$. Given $\delta \in(\hat{M})_{\text {lds }}$ and $\lambda \in \mathfrak{a}_{M, \mathbb{C}}^{*}$ we can form the representation $\delta \otimes e^{\lambda}$ of $M$ by defining

$$
\left(\delta \otimes e^{\lambda}\right)(m, a)=\lambda(\log (a)) \cdot \delta(m), \quad m \in M^{1}, a \in A_{M} .
$$

We can extend this representation trivially to $P$ and induce to $G$ in order to obtain the representation 


\section{Twisted trace Paley-Wiener theorems}

$\operatorname{ind}_{P}^{G}\left(\delta \otimes e^{\lambda}\right)$. Such induced representations may of course be formed for any parabolic subgroup of $G$, standard or not. The above induction is assumed to be normalized so that $\operatorname{ind}_{P}^{G}\left(\delta \otimes e^{\lambda}\right)$ is unitary whenever $\lambda \in i \mathfrak{a}_{M}^{*}$.

Set $K=\mathrm{SO}(n, \mathbb{R})$. It is a maximal compact subgroup of $G$. We shall adopt the compact picture of induction so that the vectors in the space of $\operatorname{ind}_{P}^{G}\left(\delta \otimes e^{\lambda}\right)$ are functions on $K$.

Suppose $x \in G$ and $H$ is a subgroup of $G$. If $\pi$ is a representation of $x^{-1} H x$ define

$$
x \pi(h)=\pi\left(x^{-1} h x\right), \quad h \in H .
$$

Similarly, if $x^{-1} M x$ is a Levi subgroup of $G$ and $\lambda \in \mathfrak{a}_{x^{-1} M x, \mathbb{C}}^{*}$ then set

$$
x \lambda(X)=\lambda\left(\operatorname{Ad}(x)^{-1}(X)\right), \quad X \in \mathfrak{a}_{M} .
$$

Here, Ad $: G \rightarrow \operatorname{GL}\left(\mathfrak{a}_{M}\right)$ is the adjoint homomorphism.

As usual we define the Weyl group $W\left(A_{M}: G\right)$ as the quotient of the normalizer of $\mathfrak{a}_{M}$ by the centralizer of $\mathfrak{a}_{M}$ with respect to the adjoint action. If $w \in W\left(A_{M}: G\right), \pi$ is a representation of $M^{1}$, and $\lambda \in \mathfrak{a}_{M, \mathbb{C}}^{*}$, then we define $w \pi$ and $w \lambda$ by choosing a representative for $w$ and following the previous scheme. Clearly, the equivalence class of $w \pi$ is independent of the choice of representative. We shall often confuse an element of a Weyl group with one of its representatives.

Suppose $H$ is a group and $h \in H$. We define the automorphism $\sigma_{h}$ of $H$ by

$$
\sigma_{h}(x)=h^{-1} x h, \quad x \in H .
$$

Given an automorphism $\sigma$ and a representation $\pi$ of $H$ set

$$
\pi^{\sigma}(x)=\pi(\sigma(x)), \quad x \in H .
$$

Notice that $\pi^{\sigma_{h}}=h \pi$ when $H=\operatorname{SL}(n, \mathbb{R})$. The representation $\pi$ is said to be $\sigma$-stable if it is equivalent to $\pi^{\sigma}$. The representation $\pi^{\sigma}$ is called the $\sigma$-conjugate of $\pi$.

The automorphism of principal interest to us is the involution $\sigma_{0}$ of $\operatorname{SL}(n, \mathbb{R})$ given by taking the transpose and inverse of a matrix. By the definition of $K=\mathrm{S} 0(n, \mathbb{R}), \sigma_{0}$ fixes $K$ pointwise. The differential of $\sigma_{0}$ is easily seen to send $X \in \mathfrak{a}_{M_{0}}$ to $-X$. This induces the map $\lambda \mapsto-\lambda$ on $\mathfrak{a}_{M_{0}, \mathbb{C}}^{*}$.

Given a real number $c>0$, we define $\lfloor c\rfloor$ to be the greatest integer less than or equal to $c$.

\section{Necessary conditions for $\sigma_{0}$-stable representations}

Suppose $P_{M}=M^{1} A_{M} U_{M}$ is a standard parabolic subgroup of $G, \rho$ is an irreducible tempered representation of $M^{1}$, and $\lambda \in \mathfrak{a}_{M, \mathbb{C}}^{*}$ with its real part lying in the open Weyl chamber of $\mathfrak{a}_{M}^{*}$ determined by $P_{M}$. The Langlands classification of irreducible admissible representations [Kna86, Theorem 8.54] tells us that $\operatorname{ind}_{P_{M}}^{G}\left(\rho \otimes e^{\lambda}\right)$ has a unique irreducible admissible quotient $J\left(P_{M}, \rho, \lambda\right)$, the Langlands quotient. Moreover, it tells us that every irreducible admissible representation of $G$ is equivalent to some Langlands quotient. As the composition of $\operatorname{ind}_{P_{M}}^{G}\left(\rho \otimes e^{\lambda}\right)$ with $\sigma_{0}$ preserves subquotients, the representation $\left(J\left(P_{M}, \rho, \lambda\right)\right)^{\sigma_{0}}$ is the unique irreducible quotient of $\left(\operatorname{ind}_{P_{M}}^{G}\left(\rho \otimes e^{\lambda}\right)\right)^{\sigma_{0}}$. In the compact picture, the vector spaces of $\left(\operatorname{ind}_{P_{M}}^{G}\left(\rho \otimes e^{\lambda}\right)\right)^{\sigma_{0}}$ and $\operatorname{ind}_{\sigma_{0} P_{M}}^{G}\left(\rho^{\sigma_{0}} \otimes e^{-\lambda}\right)$ are identical, for the elements of $K$ are fixed by $\sigma_{0}$. A simple computation shows that their actions on this vector space are also identical; that is

$$
\left(\operatorname{ind}_{P_{M}}^{G}\left(\rho \otimes e^{\lambda}\right)\right)^{\sigma_{0}}=\operatorname{ind}_{\sigma_{0} P_{M}}^{G}\left(\rho^{\sigma_{0}} \otimes e^{-\lambda}\right) .
$$

The real part of the element $-\lambda \in \mathfrak{a}_{M, \mathbb{C}}^{*}$ lies in the open Weyl chamber determined by $\sigma_{0} P_{M}$, which is opposite to $P_{M}$, so the representation on the right has a unique Langlands quotient $J\left(\sigma_{0} P_{M}, \rho^{\sigma_{0}},-\lambda\right)$. As a result, $\left(J\left(P_{M}, \rho, \lambda\right)\right)^{\sigma_{0}}$ is equal to $J\left(\sigma_{0} P_{M}, \rho^{\sigma_{0}},-\lambda\right)$. 
Now suppose that $J\left(P_{M}, \rho, \lambda\right)$ is equivalent to $\left(J\left(P_{M}, \rho, \lambda\right)\right)^{\sigma_{0}}$. Then the uniqueness statement of Langlands' classification [Lan89, Lemma 3.14] implies the existence of an element $w_{0}$, belonging to the normalizer of $A_{M}$ in $G$, such that

$$
\begin{gathered}
\sigma_{0} P_{M}=w_{0}^{-1} P_{M} w_{0}, \\
-w_{0} \lambda=\lambda, \\
w_{0}\left(\rho^{\sigma_{0}}\right) \cong \rho .
\end{gathered}
$$

If we translate $w_{0}$ on the right by an element in $M$ in this statement then the same conclusions still hold. Indeed, $M$ normalizes $\sigma_{0} P_{M}$ and is equal to the centralizer of $A_{M}$ in $G$. In other words, the conclusions depend only on the class of $w_{0}$ in $W\left(A_{M}: G\right)$.

Equation (1) implies that $P_{M}$ contains $w_{0} \sigma_{0} P_{0} w_{0}^{-1}$, as $P_{M}$ is standard. It is well-known that Borel subgroups of linear algebraic groups are conjugate. Therefore, there exists an element $m \in P_{M}$ such that

$$
m^{-1} P_{0} m=w_{0} \sigma_{0} P_{0} w_{0}^{-1} .
$$

It is easy to see that we may actually take $m$ to belong to $M$. This means that $\left(m w_{0}\right)^{-1} P_{0} m w_{0}$ is the parabolic subgroup of $G$ opposite to $P_{0}$. This fact and the invariance under $M$ described in the previous paragraph allow us to assume that $w_{0}$ is equal to the unique skew-diagonal matrix in $G$ which also lies in

$$
\left\{\left(\begin{array}{cccc}
0 & \cdots & 0 & 1 \\
\vdots & & 1 & 0 \\
0 & 1 & & \vdots \\
\pm 1 & 0 & \cdots & 0
\end{array}\right)\right\} .
$$

Having ascertained the class of $w_{0}$ in $W\left(A_{M}: G\right)$, we may compute directly from Equation (1) that the block sizes $n_{1}, \ldots, n_{\ell}$ of $M$ satisfy $n_{j}=n_{\ell+1-j}$ for $1 \leqslant j \leqslant \ell$. We may also compute directly from Equation (2) that $\lambda$ is restricted to a subspace of $\mathfrak{a}_{M, \mathbb{C}}^{*}$ of complex dimension $\lfloor(\ell+1) / 2\rfloor$.

With regard to $\rho$ we know [Kna86, Theorem 14.91] that there exists a standard parabolic subgroup $P_{L}$ contained in $P_{M}$ with Langlands decomposition $L^{1} A_{L} U_{L}$, a representation $\delta \in(\hat{L})_{\mathrm{lds}}$, and $\nu \in i\left(\mathfrak{a}_{L}^{M}\right)^{*}$ such that

$$
\rho=\operatorname{ind}_{P_{L} \cap M^{1}}^{M^{1}}\left(\delta \otimes e^{\nu}\right) .
$$

We shall use this expansion to describe equivalence (3). We do so in three steps. First, observe that the earlier argument for $\left(\operatorname{ind}_{P_{M}}^{G}\left(\rho \otimes e^{\lambda}\right)\right)^{\sigma_{0}}$ can be mimicked to conclude that $\left(\operatorname{ind}_{P_{L} \cap M^{1}}^{M^{1}}\left(\delta \otimes e^{\nu}\right)\right)^{\sigma_{0}}$ is equal to $\operatorname{ind}_{\sigma_{0} P_{L} \cap M^{1}}^{M^{1}}\left(\delta^{\sigma_{0}} \otimes e^{-\nu}\right)$.

Second, the only Levi subgroups of $G$ supporting discrete series or limit of discrete series representations are those whose blocks are of rank one or two. Observe that the involution $\sigma_{0}$ acts on $\mathrm{SL}^{ \pm}(1, \mathbb{R})=\{1,-1\}$ as the identity, and on $\mathrm{SL}^{ \pm}(2, \mathbb{R})$ as conjugation by $\left(\begin{array}{cc}0 & 1 \\ -1 & 0\end{array}\right)$. It follows that there exists a permutation matrix $w_{L} \in L^{1}$, which depends solely on the block sizes of $L$, and satisfies $\delta^{\sigma_{0}}=w_{L} \delta$. This implies that left multiplication by the operator $\delta\left(w_{L}\right)$ intertwines $\operatorname{ind}_{\sigma_{0} P_{L} \cap M^{1}}^{M^{1}}\left(\delta^{\sigma_{0}} \otimes e^{-\nu}\right)$ with $\operatorname{ind}_{\sigma_{0} P_{L} \cap M^{1}}^{M^{1}}\left(\delta \otimes e^{-\nu}\right)$.

Third, it is a simple exercise to show that the operator defined by

$$
\left(A\left(w_{0}\right) \varphi\right)(x)=\varphi\left(w_{0}^{-1} x w_{0}\right), \quad \varphi \in \operatorname{ind}_{\sigma_{0} P_{L} \cap M^{1}}^{M^{1}}\left(\delta \otimes e^{-\nu}\right),
$$

intertwines $\operatorname{ind}_{\sigma_{0} P_{L} \cap M^{1}}^{M^{1}}\left(\delta \otimes e^{-\nu}\right)$ with $\operatorname{ind}_{w_{0} \sigma_{0} P_{L} w_{0}^{-1} \cap M^{1}}^{M^{1}}\left(w_{0} \delta \otimes e^{-w_{0} \nu}\right)$.

These three equivalences taken together with (3) imply that

$$
\left.\operatorname{ind}_{P_{L} \cap M^{1}}^{M^{1}}\left(\delta \otimes e^{\nu}\right)\right)^{\sigma_{0}} \cong \operatorname{ind}_{w_{0} \sigma_{0} P_{L} w_{0}^{-1} \cap M^{1}}^{M^{1}}\left(w_{0} \delta \otimes e^{-w_{0} \nu}\right) .
$$




\section{Twisted trace Paley-Wiener theorems}

According to [Kna86, Theorem 14.91] there exists an element $w_{M} \in M$ such that $w_{M} w_{0} A_{L} w_{0}^{-1} w_{M}^{-1}$ $=A_{L},-w_{M} w_{0} \nu=\nu$, and $w_{M} w_{0} \delta=\delta$. Among other things, we have proved the following lemma.

Lemma 1. Suppose $P_{M}=M^{1} A_{M} U_{M} \supset P_{L}=L^{1} A_{L} U_{L}$ are the Langlands decompositions of standard parabolic subgroups. Suppose $\delta \in(\hat{L})_{\mathrm{lds}}$ and $\nu \in i\left(\mathfrak{a}_{L}^{M}\right)^{*}$ such that $\operatorname{ind}_{P_{L} \cap M^{1}}^{M^{1}}\left(\delta \otimes e^{\nu}\right)$ is irreducible. Finally, suppose the real part of $\lambda \in \mathfrak{a}_{M, \mathbb{C}}^{*}$ lies in the open positive Weyl chamber determined by $P_{M}$, and $J\left(P_{M}, \operatorname{ind}_{P_{L} \cap M^{1}}^{M^{1}}\left(\delta \otimes e^{\nu}\right), \lambda\right)$ is $\sigma_{0}$-stable. Then the block sizes, $n_{1}, \ldots, n_{\ell}$, of $M$ satisfy

$$
n_{j}=n_{\ell+1-j}, \quad 1 \leqslant j \leqslant \ell,
$$

$\sigma_{0} P_{M}=w_{0} P_{M} w_{0}^{-1}$, and $w_{0} \lambda=-\lambda$. Furthermore, there exists $w_{M} \in M$ such that $w_{M} w_{0} A_{L}\left(w_{M} w_{0}\right)^{-1}$ $=A_{L}, w_{M} w_{0} \delta=\delta$, and $w_{M} w_{0} \nu=-\nu$.

\section{The construction of some $\sigma_{0}$-stable representations}

We wish to produce a set of Levi subgroups and representations which furnish $\sigma_{0}$-stable representations under parabolic induction. Our approach here shall be quite concrete in that we shall provide explicit intertwining operators demonstrating $\sigma_{0}$-stability.

Suppose $P_{M}=M^{1} A_{M} U_{M} \supset P_{L}=L^{1} A_{L} U_{L}$ and $w_{0}$ are as in the conclusion of Lemma 1 . Notice that $\sigma_{0}$ sends each of these parabolic subgroups to its opposite parabolic subgroup. Conjugation by $w_{0}$ has the same effect on $P_{M}$. However, conjugation by $w_{0}$ does not necessarily send $P_{L}$ to its opposite parabolic subgroup. In fact, unless we place some restrictions on $L$, the element $w_{0}$ need not even belong to the normalizer of $L$. This suggests that we assume $L$ satisfies the conditions of the next few paragraphs.

Let us begin with the description of $L$. The subgroup $L^{1}$ is isomorphic to

$$
\left\{\left(x_{1}, \ldots, x_{k}\right) \in \mathrm{SL}^{ \pm}\left(r_{1}, \mathbb{R}\right) \times \cdots \times \mathrm{SL}^{ \pm}\left(r_{k}, \mathbb{R}\right): \prod_{j=1}^{k} \operatorname{det}\left(x_{j}\right)=1\right\},
$$

for some $r_{1}, \ldots, r_{k}=1,2$ satisfying $\sum_{j=1}^{k} r_{j}=n$. Obviously, this subgroup is completely determined by the integers $r_{1}, \ldots, r_{k}$. Suppose $0 \leqslant t \leqslant\lfloor n / 2\rfloor$ is an integer and $I_{j}$ is the identity matrix of rank $j$. If $n$ is even let $w_{t}$ be the unique element in

$$
\left\{\left(\begin{array}{ccccc}
0 & \cdots & 0 & 0 & 1 \\
0 & \cdots & 0 & 1 & 0 \\
\vdots & 0 & I_{2 t} & 0 & \vdots \\
0 & 1 & 0 & \cdots & 0 \\
\pm 1 & 0 & \cdots & 0 & 0
\end{array}\right)\right\}
$$

which belongs to $G$. If $n$ is odd let $w_{t}$ be the unique element in

$$
\left\{\left(\begin{array}{ccccc}
0 & \cdots & 0 & 0 & 1 \\
0 & \cdots & 0 & 1 & 0 \\
\vdots & 0 & I_{2 t+1} & 0 & \vdots \\
0 & 1 & 0 & \cdots & 0 \\
\pm 1 & 0 & \cdots & 0 & 0
\end{array}\right)\right\}
$$

which belongs to $G$. Note that this definition is consistent with the definition of $w_{0}$ in $\S 3$. The element $w_{t}$ is meant to fulfill the role of $w_{M} w_{0}$ as in Lemma 1.

Let $\mathcal{L}(t)$ be the set of Levi subgroups $L \supset M_{0}$ for which $L^{1}$ is of the form (4) and satisfies the following additional requirements: 
1) $w_{t} L w_{t}^{-1}=L$;

2) there is an integer $1 \leqslant s \leqslant k$ such that $\sum_{j=1}^{s} r_{j}=t$;

$3)$ at most two of the block sizes in the sequence, $r_{s+1}, \ldots, r_{k-s}$, are equal to one.

For the remainder of this section suppose that $L$ belongs to $\mathcal{L}(t)$. The element $w_{t}$ has been chosen so that $w_{t} \sigma_{0} P_{L} w_{t}^{-1}$ is a parabolic subgroup with Levi component $L$. Unfortunately, $w_{t} \sigma_{0} P_{L} w_{t}^{-1}$ might not be a standard parabolic subgroup. After all, $w_{t}$ does nothing at all to the 'middle' blocks of $L$, whereas $\sigma_{0}$ sends $P_{L}$ to its opposite parabolic subgroup. To correct this discrepancy, we shall eventually turn to some intertwining operators of Knapp and Stein. Before this, we present the relevant parabolic subgroups.

In keeping with the earlier definitions, we specify a Levi subgroup $M_{L, t} \supset L$ by referring to its block sizes $r_{1}^{\prime}, \ldots, r_{k^{\prime}}^{\prime}$. Working under the assumptions of the previous paragraph, set $M_{L, t}=L$ if $k=2 s$. Otherwise set

$$
r_{j}^{\prime}=r_{j}, 1 \leqslant j \leqslant s, \quad r_{s+1}^{\prime}=\sum_{j=s+1}^{k-s} r_{j}, \quad r_{2 s+2-j}^{\prime}=r_{j}^{\prime}=r_{j}, 1 \leqslant j \leqslant s .
$$

Clearly, the standard parabolic subgroup $P_{M_{L, t}}=M_{L, t}^{1} A_{M_{L, t}} U_{M_{L, t}}$ contains $P_{L}$. It is easy to see that $U_{M_{L, t}}$ is contained in $U_{L}$, and it is left as an exercise to the reader to show that

$$
w_{t} \sigma_{0} P_{L} w_{t}^{-1}=\left(\sigma_{0} P_{L} \cap M_{L, t}\right) U_{M_{L, t}} .
$$

We now list sets of representations that are attached to $L \in \mathcal{L}(t)$ and $w_{t}$ in terms of Weyl groups. Let $\mathfrak{g}$ be the Lie algebra of $G$. The set of useful roots $\Delta_{L}$ of $\left(\mathfrak{g}, \mathfrak{a}_{L}\right)$ forms a root system [Kna86, Theorem 14.39]. We fix a set of positive roots $\Delta_{L}^{+}$with respect to the parabolic subgroup $P_{L}$. Given an irreducible tempered representation $\delta$ of $L^{1}$, set $W_{\delta}$ to be the subgroup of the Weyl group of $\Delta_{L}$ which stabilizes the equivalence class of $\delta$. The group $W_{\delta}$ is the semidirect product of two abstract Weyl groups [Kna86, ch. XIV, § 9]. One of them is the R group $R_{\delta}$ and is, as we shall soon see, isomorphic to $\mathbb{Z} / 2 \mathbb{Z}$. The $\mathrm{R}$ group normalizes the other subgroup $W_{\delta}^{0} \subset W_{\delta}$, which is the Weyl group of a root system $\Delta_{\delta}^{0} \subset \Delta_{L}$. We fix the set of positive roots of $\Delta_{\delta}^{0}$ to be $\Delta_{\delta}^{0} \cap \Delta_{L}^{+}$.

By applying a well-known property of Weyl groups [Kna86, ch. IV, § 4, property (2)], one can show that the subgroup $Z_{W_{\delta}}\left(R_{\delta}\right)$ of elements in $W_{\delta}$ which are centralized by $R_{\delta}$ is an abstract Weyl group. In fact, its root system is the direct product of the root system of $R_{\delta}$ and the subset of roots in $\Delta_{\delta}^{0}$ which are fixed by $R_{\delta}$. If $R_{\delta}$ is non-trivial then $\Delta_{L}^{+}$determines a unique positive root $\alpha_{R}$ in the root system of $R_{\delta}$ [Kna86, Theorem 14.64]. We may therefore fix a set of positive roots for the above direct product by taking the union of $\alpha_{R}$ with the intersection of $\Delta_{L}^{+}$and the second root system in the product.

Define $(\hat{L})_{\mathrm{lds}, t}$ to be the subset of $(\hat{L})_{\mathrm{lds}}$ given by those $\delta \in(\hat{L})_{\mathrm{lds}}$ such that $w_{t}$ is (a representative of) the longest element in either $W_{\delta}^{0}$ or $Z_{W_{\delta}}\left(R_{\delta}\right)$.

Given an element $w \in W\left(A_{L}: G\right)$, define $\mathfrak{a}_{L}^{w}$ to be the subspace of $\mathfrak{a}_{L}$ spanned by the elements $X \in \mathfrak{a}_{L}$ which satisfy $\operatorname{Ad}(w)(X)=-X$. We denote the relevant dual spaces and their complexifications as we do for $\mathfrak{a}_{L}$.

Proposition 1. Suppose $\delta$ is (a representative of a class) in $(\hat{L})_{\mathrm{lds}, t}$ and $\nu \in\left(\mathfrak{a}_{L, \mathbb{C}}^{w_{t}}\right)^{*}$. Then $\operatorname{ind}_{P_{L}}^{G}(\delta \otimes$ $\left.e^{\nu}\right)$ is $\sigma_{0}$-stable.

Proof. We shall define an operator $T(\delta)$ intertwining $\left(\operatorname{ind}_{P_{L}}^{G}\left(\delta \otimes e^{\nu}\right)\right)^{\sigma_{0}}$ with $\operatorname{ind}_{P_{L}}^{G}\left(\delta \otimes e^{\nu}\right)$. As in earlier considerations, we see that $\left(\operatorname{ind}_{P_{L}}^{G}\left(\delta \otimes e^{\nu}\right)\right)^{\sigma_{0}}$ is equal to $\operatorname{ind}_{\sigma_{0} P_{L}}^{G}\left(\delta^{\sigma_{0}} \otimes e^{-\nu}\right)$. The latter representation is equivalent to

$$
\operatorname{ind}_{w_{t} \sigma_{0} P_{L} w_{t}^{-1}}^{G}\left(w_{t}\left(\delta^{\sigma_{0}}\right) \otimes e^{-w_{t} \nu}\right)=\operatorname{ind}_{w_{t} \sigma_{0} P_{L} w_{t}^{-1}}^{G}\left(w_{t}\left(\delta^{\sigma_{0}}\right) \otimes e^{\nu}\right)
$$




\section{Twisted trace Paley-Wiener theorems}

by virtue of the intertwining operator

$$
\left(A\left(w_{t}\right) \varphi\right)(x)=\varphi\left(w_{t}^{-1} x w_{t}\right), \quad \varphi \in \operatorname{ind}_{\sigma_{0} P_{L}}^{G}\left(\delta^{\sigma_{0}} \otimes e^{-\nu}\right) .
$$

The intertwining operator $\delta\left(w_{L}\right)$ (which depends only on $L$ ) defined in $\S 3$ intertwines the representation on the right with

$$
\operatorname{ind}_{w_{t} \sigma_{0} P_{L} w_{t}^{-1}}^{G}\left(w_{t} \delta \otimes e^{\nu}\right) .
$$

As $w_{t} \delta \cong \delta$, [Kna86, Theorem 14.91] provides an element $w_{\delta} \in L \cap K$ such that left multiplication by $\delta\left(w_{\delta}\right)$ intertwines the above representation with

$$
\text { ind }_{w_{t} \sigma_{0} P_{L} w_{t}^{-1}}^{G}\left(\delta \otimes e^{\nu}\right) .
$$

Knapp and Stein have defined a normalized intertwining operator

$$
\mathcal{A}\left(P_{L}: w_{t} \sigma_{0} P_{L} w_{t}^{-1}: \delta: \nu\right)
$$

which intertwines $\operatorname{ind}_{w_{t} \sigma_{0} P_{L} w_{t}^{-1}}^{G}\left(\delta \otimes e^{\nu}\right)$ with $\operatorname{ind}_{P_{L}}^{G}\left(\delta \otimes e^{\nu}\right)$, for $\nu$ in a dense open subset of $\left(\mathfrak{a}_{L, \mathbb{C}}^{M}\right)^{*}$ $[$ Kna $86, \S 6, \mathrm{XIV}]$. It follows from Equation (5) and the inductive definition of this operator that it is induced from an intertwining operator

$$
\mathcal{A}\left(P_{L} \cap M_{L, t}^{1}: \sigma_{0} P_{L} \cap M_{L, t}^{1}: \delta: \nu_{M_{L, t}}\right),
$$

where $\nu_{M_{L, t}}$ is the orthogonal projection of $\nu$ to $\left(\mathfrak{a}_{L, \mathbb{C}}^{M_{L, t}}\right)^{*}$. It is an immediate consequence of the definitions that

$$
w_{t} \nu_{1}=\nu_{1}, \quad \nu_{1} \in\left(\mathfrak{a}_{L, \mathbb{C}}^{M_{L, t}}\right)^{*} .
$$

From this equation and the fact that $w_{t} \nu=-\nu$ it follows that $\nu_{M_{L, t}}=0$. By [Kna86, Proposition 14.20(d)], the operator $\mathcal{A}\left(P_{L} \cap M_{L, t}^{1}: \sigma_{0} P_{L} \cap M_{L, t}^{1}: \delta: 0\right)$ is defined. We may therefore use it to intertwine representation (6) with $\operatorname{ind}_{P_{L}}^{G}\left(\delta \otimes e^{\nu}\right)$. For convenience, we shall identify $\mathcal{A}\left(P_{L} \cap M_{L, t}^{1}\right.$ : $\left.\sigma_{0} P_{L} \cap M_{L, t}^{1}: \delta: 0\right)^{-1}$ with its corresponding induced operator on $\operatorname{ind}_{w_{t} \sigma_{0} P_{L} w_{t}^{-1}}^{G}\left(\delta \otimes e^{\nu}\right)$.

After working through these equivalences in reverse, we find that

$$
T(\delta)=\mathcal{A}\left(P_{L} \cap M_{L, t}^{1}: \sigma_{0} P_{L} \cap M_{L, t}^{1}: \delta: 0\right)^{-1} \delta\left(w_{\delta}^{-1}\right) \delta\left(w_{L}^{-1}\right) A\left(w_{t}^{-1}\right)
$$

is an intertwining operator between $\operatorname{ind}_{P_{L}}^{G}\left(\delta \otimes e^{\nu}\right)$ and $\left(\operatorname{ind}_{P_{L}}^{G}\left(\delta \otimes e^{\nu}\right)\right)^{\sigma_{0}}$.

We can simplify the notation in the definition of $T(\delta)$ somewhat by defining operators $\mathrm{L}(w)$ and $\mathrm{R}(w)$ on $\varphi \in \operatorname{ind}_{P_{L}}^{G}\left(\delta \otimes e^{\nu}\right)$ by

$$
(\mathrm{L}(w) \varphi)(x)=\varphi\left(w^{-1} x\right), \quad(\mathrm{R}(w) \varphi)(x)=\varphi(x w), \quad w \in K .
$$

Unraveling the definitions, and noting that

$$
\mathcal{A}\left(P_{L} \cap M_{L, t}: \sigma_{0} P_{L} \cap M_{L, t}: \delta: 0\right)^{-1}=\mathcal{A}\left(\sigma_{0} P_{L} \cap M_{L, t}: P_{L} \cap M_{L, t}: \delta: 0\right)
$$

[Kna86, Lemma 14.18], we see that $T(\delta)$ reduces to

$$
T(\delta)=\mathcal{A}\left(\sigma_{0} P_{L} \cap M_{L, t}: P_{L} \cap M_{L, t}: \delta: 0\right) \mathrm{L}\left(w_{\delta}^{-1} w_{L}^{-1} w_{t}\right) \mathrm{R}\left(w_{t}^{-1}\right) .
$$

It is valuable to note that $T(\delta)$ is independent of $\nu \in\left(\mathfrak{a}_{L, \mathbb{C}}^{w_{t}}\right)^{*}$.

\section{The exhaustion of the $\sigma_{0}$-stable representations}

Our goal here is to show that every irreducible $\sigma_{0}$-stable admissible representation is equivalent to a quotient of $\operatorname{ind}_{P_{L}}^{G}\left(\delta^{\prime} \otimes e^{\lambda^{\prime}}\right)$, where $L \in \mathcal{L}(t), \delta^{\prime} \in\left(\hat{L}^{\prime}\right)_{\mathrm{lds}, t}$, and $\lambda^{\prime} \in\left(\mathfrak{a}_{L^{\prime}, \mathbb{C}}^{w_{t}}\right)^{*}$ for some $0 \leqslant t \leqslant$ $\lfloor n / 2\rfloor$. This is not a classification of the irreducible $\sigma_{0}$-stable representations for we have provided neither a uniqueness assertion nor a description of the quotients of $\operatorname{ind}_{P_{L}}^{G}\left(\delta^{\prime} \otimes e^{\lambda^{\prime}}\right)$. These additional 


\section{P. Mezo}

features may be derived from a careful application of the Langlands classification and [Kna86, Theorem 14.91]. (For a better impression of these issues, consult the example at the beginning of $\S 6$.) In any case, the characters of irreducible $\sigma_{0}$-stable representations may be recovered in a prescribed fashion from the characters of the representations $\operatorname{ind}_{P_{L}}^{G}\left(\delta^{\prime} \otimes e^{\lambda^{\prime}}\right)$ as above (cf. [Art89, $\S 5])$.

In light of Lemma 1, we will achieve the above goal if we prove the following proposition.

Proposition 2. Suppose $P_{M}, P_{L}, \delta$, and $\nu$ are as in the conclusion of Lemma 1. Set $n^{\prime}=n_{(\ell+1) / 2}$ if $\ell$ is odd and $n^{\prime}=0$ otherwise. Then there exist $0 \leqslant t \leqslant n^{\prime}, L^{\prime} \in \mathcal{L}(t), \delta^{\prime} \in\left(\hat{L}^{\prime}\right)_{\operatorname{lds}, t}$, and $\nu^{\prime} \in i\left(\mathfrak{a}_{L^{\prime}}^{w_{t}}\right)^{*}$ such that $P_{L^{\prime}} \subset P_{M}$ and $\operatorname{ind}_{P_{L} \cap M^{1}}^{M^{1}}\left(\delta \otimes e^{\nu}\right)$ is equivalent to $\operatorname{ind}_{P_{L^{\prime}} \cap M^{1}}^{M^{1}}\left(\delta^{\prime} \otimes e^{\nu^{\prime}}\right)$.

Indeed, the integer $n^{\prime}$ of this proposition has been chosen so that $w_{t} \lambda=w_{0} \lambda$ whenever $\lambda \epsilon$ $\left(\mathfrak{a}_{M, \mathbb{C}}^{w_{0}}\right)^{*}$. Therefore, one may take the $\lambda^{\prime}$ occurring in the above representation $\operatorname{ind}_{P_{L}}^{G}\left(\delta^{\prime} \otimes e^{\lambda^{\prime}}\right)$ equal to $\lambda+\nu^{\prime} \in\left(\mathfrak{a}_{L^{\prime}, \mathbb{C}}^{w_{t}}\right)^{*}$. The proof of this proposition will consume this entire section.

Proof of Proposition 2. Suppose that $w \in M$ permutes the blocks of $L$. Then the operator

$$
S(w, \delta, \nu)=\mathcal{A}\left(P_{w L w^{-1}} \cap M^{1}: w P_{L} w^{-1} \cap M^{1}: w \delta: w \nu\right) L(w)
$$

(cf. $\S 4$ with apologies for the double usage of $L$ ) is invertible and intertwines $\operatorname{ind}_{P_{L}}^{G}\left(\delta \otimes e^{\nu}\right)$ with $\operatorname{ind}_{P}^{G}{ }_{w L-1}\left(w \delta \otimes e^{w \nu}\right)$ for any $\nu \in i\left(\mathfrak{a}_{L}\right)^{*}[$ Kna86, Proposition 14.20(d)]. The upshot of this observation is that in order to prove Proposition 2 it suffices to find an element $w \in M$ which permutes the blocks of $L$ such that the following properties are satisfied:

i) $w L w^{-1}=L^{\prime}$ for some $L^{\prime} \in \mathcal{L}(t), 0 \leqslant t \leqslant n^{\prime}$;

ii) $w \delta \in\left(\hat{L}^{\prime}\right)_{\mathrm{lds}, t}$;

iii) $w \nu \in i\left(\mathfrak{a}_{L^{\prime}}^{w_{t}}\right)^{*}$.

We shall prove these three properties by portraying $\delta$ as a $k$-tuple of representations of $\mathrm{SL}^{ \pm}\left(r_{j}, \mathbb{R}\right)$, $r_{j}=1,2$, and providing permutations of these representations which will also place $\delta$ and $\nu$ into the desired form. Suppose $\delta$ and $\nu$ are as in Lemma 1 and $L^{1}$ is of the form (4). Then there exists an irreducible representation $\delta^{ \pm}$of

$$
L^{ \pm} \cong \mathrm{SL}^{ \pm}\left(r_{1}, \mathbb{R}\right) \times \cdots \times \mathrm{SL}^{ \pm}\left(r_{k}, \mathbb{R}\right),
$$

whose restriction $\delta_{\mid L^{1}}^{ \pm}$to $L^{1}$ contains $\delta$ as one of its two possible subrepresentations. Indeed, by Frobenius reciprocity $\delta^{ \pm}$can be taken to be an irreducible subrepresentation of the induced representation of $\delta$ to $L^{ \pm}$. Clearly, we may express $\delta^{ \pm}$uniquely as a $k$-tuple $\left(\left(\delta^{ \pm}\right)_{1}, \ldots,\left(\delta^{ \pm}\right)_{k}\right)$ of irreducible representations of $\mathrm{SL}^{ \pm}\left(r_{1}, \mathbb{R}\right) \times \cdots \times \mathrm{SL}^{ \pm}\left(r_{k}, \mathbb{R}\right)$. It is important to note that there may be another inequivalent representation of $L^{ \pm}$whose restriction to $L^{1}$ contains $\delta$. The following lemma fully describes this possibility in terms of the non-trivial character $\operatorname{sgn}$ of $\operatorname{SL}^{ \pm}(1, \mathbb{R})=\{1,-1\}$.

Lemma 2. Suppose $\delta^{ \pm}=\left(\left(\delta^{ \pm}\right)_{1}, \ldots,\left(\delta^{ \pm}\right)_{k}\right)$ and $\delta^{ \pm}=\left(\left(\delta^{ \pm}\right)_{1}, \ldots,\left(\delta^{\prime \pm}\right)_{k}\right)$ are irreducible representations of $L^{ \pm}$such that $\delta_{\mid L^{1}}^{ \pm}$and $\delta_{\mid L^{1}}^{\prime \pm}$ contain $\delta$ as a subrepresentation. Then $\delta_{j}^{ \pm}=\delta_{j}^{\prime \pm}$, for $1 \leqslant j \leqslant k$ such that $r_{j}=2$. Furthermore, either $\left(\delta^{ \pm}\right)_{j}=\left(\delta^{ \pm}\right)_{j}$, for all $1 \leqslant j \leqslant k$ such that $r_{j}=1$, or $\left(\delta^{ \pm}\right)_{j}=\operatorname{sgn} \cdot\left(\delta^{\prime \pm}\right)_{j}$, for all $1 \leqslant j \leqslant k$ such that $r_{j}=1$.

Proof. Suppose $1 \leqslant j \leqslant k$ and $r_{j}=2$. Then, according to (and in the notation of) [Kna79, $\S 2$ ], there exist positive integers $d_{j}, d_{j}^{\prime}$ such that $\left(\delta^{ \pm}\right)_{j}$ and $\left(\delta^{\prime \pm}\right)_{j}$ are the respective induced representations of $\mathcal{D}_{d_{j}}^{+}$and $\mathcal{D}_{d_{j}^{\prime}}^{+}$. Moreover, we have $\left(\left(\delta^{ \pm}\right)_{j}\right)_{\mid \mathrm{SL}(2, \mathbb{R})}=\mathcal{D}_{d_{j}}^{+} \oplus \mathcal{D}_{d_{j}}^{-}$and $\left(\left(\delta^{\prime \pm}\right)_{j}\right)_{\mid \mathrm{SL}(2, \mathbb{R})}=\mathcal{D}_{d_{j}^{\prime}}^{+} \oplus \mathcal{D}_{d_{j}^{\prime}}^{-}$. 


\section{Twisted trace Paley-Wiener theorems}

It follows that $d_{j}=d_{j}^{\prime}$ and the first assertion of the lemma is proven. Now set

$$
H=\left\{\left(x_{1}, \ldots, x_{k}\right) \in L^{1}: x_{j}=1, \text { if } r_{j}=2, \text { and } \prod_{j=1}^{k} \operatorname{det}\left(x_{j}\right)=1\right\} .
$$

It is a simple exercise to show that if the one-dimensional representation $\delta_{\mid H}^{ \pm}$is trivial then either $\left(\delta^{ \pm}\right)_{j}$ is the trivial character for all $1 \leqslant j \leqslant k$ such that $r_{j}=1$, or $\left(\delta^{ \pm}\right)_{j}=$ sgn for all $1 \leqslant j \leqslant k$ such that $r_{j}=1$. The lemma now follows by replacing $\delta_{\mid H}^{ \pm}$in this exercise with $\delta_{\mid H}^{ \pm}\left(\delta_{\mid H}^{\prime \pm}\right)^{-1}$.

We are assuming that $w_{M} w_{0} \delta \cong \delta$ as in Lemma 1. This implies that $w_{M} w_{0} \delta_{\mid L^{1}}^{ \pm}$contains $\delta$ as a subrepresentation. Therefore, Lemma 2 tells us that either $w_{M} w_{0} \delta^{ \pm}$is equivalent to $\delta^{ \pm}$, or both the trivial and sign characters of $\mathrm{SL}^{ \pm}(1, \mathbb{R})$ occur in $\left(\left(\delta^{ \pm}\right)_{1}, \ldots,\left(\delta^{ \pm}\right)_{k}\right)$ an equal number of times and $w_{M} w_{0}$ sends each trivial character to a sign character. We shall prove the three properties (i)-(iii) by considering each of these two cases separately.

First we fix some more notation for the representations occurring in $\delta^{ \pm}$. Let $\tau_{1}, \ldots, \tau_{b}$ be mutually inequivalent representations of $\mathrm{SL}^{ \pm}(2, \mathbb{R})$ or $\mathrm{SL}^{ \pm}(1, \mathbb{R})$ such that every representation occurring in the expansion $\left(\left(\delta^{ \pm}\right)_{1}, \ldots,\left(\delta^{ \pm}\right)_{k}\right)$ of $\delta^{ \pm}$is equivalent to some $\tau_{j}, 1 \leqslant j \leqslant b$, and vice versa. Let $a_{j}$ be the number of representations in $\left(\left(\delta^{ \pm}\right)_{1}, \ldots,\left(\delta^{ \pm}\right)_{k}\right)$ which are equivalent to $\tau_{j}, 1 \leqslant j \leqslant b$.

We now prove properties (i)-(iii) under the assumption that $w_{M} w_{0} \delta^{ \pm}$is equivalent to $\delta^{ \pm}$. Recall that $M$ is comprised of $\ell$ blocks. We define $M^{ \pm} \supset L^{ \pm}$in the obvious way. If $\left(\delta^{ \pm}\right)_{j}$ is a representation of a block of $L^{ \pm}$contained in the $i$ th block of $M^{ \pm}$, then $w_{M} w_{0} \delta^{ \pm} \cong \delta^{ \pm}$implies that it also occurs as a representation of a block of $L^{ \pm}$contained in the $(\ell+1-i)$ th block of $M^{ \pm}$. It follows that when $\ell$ is even the integers $a_{1}, \ldots, a_{b}$ are all even and that there exists $w \in M^{1}$ which permutes the blocks of $L$ such that $w_{0} w \delta^{ \pm} \cong w \delta^{ \pm}$.

On the other hand, suppose that $\ell$ is odd. Let $t$ be the number of integers in $a_{1}, \ldots, a_{b}$ which are odd. Using the same reasoning as in the case that $\ell$ is even, it is not difficult to see that there exists $w \in M^{1}$ which permutes the blocks of $L$ in such a way that each representation of $\tau_{1}, \ldots, \tau_{b}$ occurring an odd number of times in $\left(\left(\delta^{ \pm}\right)_{1}, \ldots,\left(\delta^{ \pm}\right)_{k}\right)$ occurs an odd number of times as a representation of a block contained in $M_{(\ell+1) / 2}$. In particular, the integer $t$ is no greater than the block size $n_{(\ell+1) / 2}$. Moreover, we may assume that $w$ satisfies $w_{t} w \delta^{ \pm}=w \delta^{ \pm}$.

Bearing in mind our observation concerning the intertwining operator (8) and permutations of the blocks of $L$, we may now assume that $\delta^{ \pm}$satisfies $w_{t} \delta^{ \pm}=\delta^{ \pm}$for some $0 \leqslant t \leqslant n^{\prime}$. This implies that $L \in \mathcal{L}(t)$ and that (the class of) $w_{t}$ belongs to $W_{\delta}$ by restricting $\delta^{ \pm}$to $L^{1}$. That is to say, we may assume that property (i) in our proof holds with $L=L^{\prime}$.

To obtain property (ii) we wish to show that $w_{t}$ is (a representative of) the longest element in $W_{\delta}^{0}$. For this we require a better understanding of $R_{\delta}$. Apparently, the Weyl group $W\left(A_{L}: G\right)$ is the direct product of the permutation group $W_{e}$, of the blocks of rank two occurring in $L$, and the permutation group $S$, of the blocks of rank one occurring in $L$. (The subgroup $W_{e}$ is generated by reflections of the even roots of $\Delta_{L}$ (for definitions see [Kna86, § 10, XIV]).) [Kna86, Corollary 14.50] and [Kna86, Theorem 14.59] together imply that $R_{\delta}$ is contained in $S$. In view of Lemma 2, an element $\tilde{w} \in S \cap W_{\delta}$ can have either one of two possible effects: either it sends the trivial characters of $\delta^{ \pm}$to trivial characters and the sign characters of $\delta^{ \pm}$to sign characters; or the trivial and sign characters of $\delta^{ \pm}$appear an equal (positive) number of times, and $\tilde{w}$ sends each trivial character to a sign character and vice versa. We can reduce the question of whether $\tilde{w}$ lies in $R_{\delta}$ to the case $G=\mathrm{SL}(2, \mathbb{R})$ by applying [Kna86, Theorem 14.43(b)]. Combining this theorem with our knowledge of $\mathrm{R}$ groups for $\operatorname{SL}(2, \mathbb{R})$, we can deduce that $\tilde{w}$ lies in $R_{\delta}$ only if the second possibility for $\tilde{w}$ holds. As $w_{t} \delta^{ \pm} \cong \delta^{ \pm}$, the second possibility does not hold for the action of $w_{t}$ on the blocks of rank one. Therefore, $w_{t}$ belongs to $W_{\delta}^{0}$. In addition, $W_{\delta}^{0}$ is isomorphic to $W_{1} \times \cdots \times W_{b}$, where $W_{j}$ is the 
permutation group of the representations in $\left(\left(\delta^{ \pm}\right)_{1}, \ldots,\left(\delta^{ \pm}\right)_{k}\right)$ equivalent to $\tau_{j}, 1 \leqslant j \leqslant b$. It follows that the simple reflections of $W_{\delta}^{0}$ are those which transpose the $i$ th block of $L$ with the $j$ th block, where $j$ is the least number greater than $i$ such that $\left(\delta^{ \pm}\right)_{i} \cong\left(\delta^{ \pm}\right)_{j}$. The action of $w_{t}$ on $\Delta_{\delta}^{0}$ sends each simple root to a negative root. The longest element of $W_{\delta}^{0}$ is therefore equal to (the class of) $w_{t}$ [Hum72, Lemma 10.3A]. This completes property the proof of property (ii)

To achieve property (iii) we require some more information about $w_{M} w_{0}$. Since we are assuming that $w_{M} w_{0}$ fixes $\delta^{ \pm}$, we know from our description of $W_{\delta}$ that it belongs to $W_{\delta}^{0}$. We may therefore suppose that $\nu \in i\left(\mathfrak{a}_{L}^{M}\right)^{*}$ such that $w \nu=-\nu$ for $w=w_{M} w_{0} \in W_{\delta}^{0}$. Our goal is to show that there exists $w^{\prime} \in W_{\delta}^{0}$ such that $w_{t} w^{\prime} \nu=-w^{\prime} \nu$, that is, that $w^{\prime} \nu \in i\left(\mathfrak{a}_{L}^{w_{t}}\right)^{*}$; for then we may set $\delta^{\prime}=w^{\prime} \delta=\delta, \nu^{\prime}=w^{\prime} \nu$, and the conditions of Proposition 2 are fulfilled. We may choose $w^{\prime}$ to be an element of $W_{\delta}^{0}$ such that $w^{\prime} \nu$ lies in the closure of the positive Weyl chamber determined by $\Delta_{\delta}^{0} \cap \Delta_{L}^{+}$. After all, $W_{\delta}^{0}$ acts simply transitively on the Weyl chambers, and our assumption that $w \in W_{\delta}^{0}$ implies that $\nu$ is in the real linear span of $i \Delta_{\delta}^{0}$. The element $w_{t}\left(w^{\prime} w\left(w^{\prime}\right)^{-1}\right) w^{\prime} \nu$ is also in the closure of the positive Weyl chamber, as $\left(w^{\prime} w\left(w^{\prime}\right)^{-1}\right) w^{\prime} \nu=-w^{\prime} \nu$ lies in the closure of the chamber opposite to the positive chamber and $w_{t}$ sends this latter chamber back to the positive one. By [Hum72, Lemma 10.3B], we have $w_{t}\left(w^{\prime} w\left(w^{\prime}\right)^{-1}\right) w^{\prime} \nu=w^{\prime} \nu$, whence

$$
w_{t} w^{\prime} \nu=\left(w_{t}\right)^{-1} w^{\prime} \nu=\left(w^{\prime} w\left(w^{\prime}\right)^{-1}\right) w^{\prime} \nu=-w^{\prime} \nu
$$

and property (iii) is satisfied.

We now prove Proposition 2 in the case that $w_{M} w_{0} \delta \cong \delta$, but $w_{M} w_{0} \delta^{ \pm}$is not equivalent to $\delta^{ \pm}$. According to our remarks concerning the nature of the $\mathrm{R}$ group, this implies that $R_{\delta}$ is non-trivial and that (the class of) $w_{M} w_{0}$ does not belong to $W_{\delta}^{0}$. We have also pointed out that $R_{\delta} \neq\{1\}$ implies that the trivial and sign characters of $\mathrm{SL}^{ \pm}(1, \mathbb{R})$ appear in $\left(\left(\delta^{ \pm}\right)_{1}, \ldots,\left(\delta^{ \pm}\right)_{k}\right)$ and that they do so an equal number of times. We shall assume that $\tau_{b-1}$ is the trivial character and $\tau_{b}$ is the sign character, so that $a_{b-1}=a_{b}$. Our assumption and Lemma 2 imply that if $\left(\delta^{ \pm}\right)_{j}$ is a representation of a rank-two block of $L^{ \pm}$contained in the $i$ th block of $M^{ \pm}$, then it also occurs as a representation of a block of $L^{ \pm}$contained in the $(\ell+1-i)$ th block of $M^{ \pm}$. In contrast, if $\left(\delta^{ \pm}\right)_{j}$ is a representation of a rank-one block of $L^{ \pm}$contained in the $i$ th block of $M^{ \pm}$, then $\operatorname{sgn} \cdot\left(\delta^{ \pm}\right)_{j}$ occurs as a representation of a block of $L^{ \pm}$contained in the $(\ell+1-i)$ th block of $M^{ \pm}$. Let $t$ be the number of representations among $\tau_{1}, \ldots, \tau_{b}$ which are not representations of $\mathrm{SL}^{ \pm}(1, \mathbb{R})$ and which appear an odd number of times in $\left(\left(\delta^{ \pm}\right)_{1}, \ldots,\left(\delta^{ \pm}\right)_{k}\right)$. It should be clear that there is an element $w \in M^{1}$, permuting the blocks of $L$, such that

$$
\left(w_{t} w \delta^{ \pm}\right)_{j}= \begin{cases}\left(w \delta^{ \pm}\right)_{j}, & \text { if }\left(w \delta^{ \pm}\right)_{j} \text { is a representation of } \mathrm{SL}^{ \pm}(2, \mathbb{R}) \\ \operatorname{sgn} \cdot\left(w \delta^{ \pm}\right)_{j}, & \text { otherwise }\end{cases}
$$

for all $1 \leqslant j \leqslant k$.

As in the previous case, we may now assume that $L \in \mathcal{L}(t)$ and $w_{t} \delta=\delta$, so that property (i) holds with $L=L^{\prime}$. We wish to show property (ii) by proving that $w_{t}$ may be taken to be (a representative of) the longest element in $Z_{W_{\delta}}\left(R_{\delta}\right)$. Let us examine the structure of $R_{\delta}$ is some more detail. Recall that a non-trivial element $r$ of $R_{\delta}$ sends each trivial character of $\delta^{ \pm}$to a sign character of $\delta^{ \pm}$and vice versa. Moreover, it leaves the remaining representations occurring in $\left(\left(\delta^{ \pm}\right)_{1}, \ldots,\left(\delta^{ \pm}\right)_{k}\right)$ unaffected. By definition, $r$ also stabilizes the set $\Delta_{\delta}^{0} \cap \Delta_{L}^{+}$. Consequently, there is an isomorphism between $W_{\delta}$ and

$$
W_{1} \times \cdots \times W_{b-2} \times\left(\left(W_{b-1} \times W_{b}\right) \rtimes R_{\delta}\right)
$$

in which $r$ acts on $W_{b-1} \times W_{b}$ by transposition. In particular, $R_{\delta}$ is isomorphic to $\mathbb{Z} / 2 \mathbb{Z}$. The longest element of $Z_{W_{\delta}}\left(R_{\delta}\right)$ is seen to be the product of $r$ and the longest element of $W_{\delta}^{0}$. This element is distinguished by the property that it sends each positive simple root to a negative root and that it transposes each trivial representation in $\delta^{ \pm}$with a sign representation. According to (9), $w_{t}$ has 


\section{TWisted trace Paley-Wiener theorems}

the latter property. The former property is deduced for $w_{t}$ just as in the case that $w_{M} w_{0} \delta^{ \pm} \cong \delta^{ \pm}$. Consequently, $w_{t}$ is the longest element in $Z_{W_{\delta}}\left(R_{\delta}\right)$.

We have just shown that property (ii) in the proof of Proposition 2 is satisfied. To obtain property (iii), we may suppose that $\nu \in i\left(\mathfrak{a}_{L}^{M}\right)^{*}$ such that $r w \nu=-\nu$ for some $w \in W_{\delta}^{0}$. In the notation of $\S 4$, this equation implies that there exist $\nu_{0}$ in the real linear span of $i \Delta_{\delta}^{0}$, and $\nu_{R}$, a real multiple of $i \alpha_{R}$, such that $\nu=\nu_{0}+\nu_{R}$. Since the elements of $\Delta_{\delta}^{0}$ are orthogonal to $\alpha_{R}$, this decomposition is unique and elements of $W_{\delta}^{0}$ act as the identity on $\nu_{R}$. Once again, we aim to prove Proposition 2 by showing that there exists $w^{\prime} \in W_{\delta}^{0}$ such that $w_{t} w^{\prime} \nu=-w^{\prime} \nu$. We choose $w^{\prime} \in W_{\delta}^{0}$ such that $w^{\prime} \nu_{0}$ lies in the closure of the positive Weyl chamber determined by $\Delta_{\delta}^{0} \cap \Delta_{L}^{+}$. The element $\left(w^{\prime} r w\left(w^{\prime}\right)^{-1}\right) w^{\prime} \nu_{0}=-w^{\prime} \nu_{0}$ clearly lies in the closure of the opposite Weyl chamber. It is then immediate from the definition of $R_{\delta}$ that $r\left(w^{\prime} r w\left(w^{\prime}\right)^{-1}\right) w^{\prime} \nu_{0}$ lies in the closure of the opposite chamber as well. It is readily verified that $w_{t} r$ is the longest element of $W_{\delta}^{0}$, and so the element

$$
w_{t}\left(w^{\prime} r w\left(w^{\prime}\right)^{-1}\right) w^{\prime} \nu_{0}=\left(w_{t} r\right)\left(r\left(w^{\prime} r w\left(w^{\prime}\right)^{-1}\right)\right) w^{\prime} \nu_{0}
$$

belongs once again to the closure of the positive chamber. [Hum72, Lemma 10.3B] then implies that $w_{t}\left(w^{\prime} r w\left(w^{\prime}\right)^{-1}\right) w^{\prime} \nu_{0}=w^{\prime} \nu_{0}$ and in turn that $w_{t} w^{\prime} \nu_{0}=-w^{\prime} \nu_{0}$. Therefore

$$
w_{t} w^{\prime} \nu=-w^{\prime} \nu_{0}+\left(w_{t} r\right) r \nu_{R}=-w^{\prime} \nu_{0}-\nu_{R}=-w^{\prime} \nu
$$

and property (iii) is satisfied. This concludes the proof of Proposition 2.

\section{Some more intertwining operators}

Proposition 2 gives us a method of associating any $\sigma_{0}$-stable representation to one of the representations in Proposition 1 by way of a permutation matrix. We shall use this method to define intertwining operators for any $\delta \in(\hat{L})_{\text {lds }}$ and Levi subgroup $L \supset M_{0}$. In order to apply the existing trace Paley-Wiener theorems to our context it is important that these intertwining operators satisfy two properties.

The first property is one of compatibility for representations which are affiliated by induction. More precisely, let

$$
P_{L_{i}}=L_{i}^{1} A_{L_{i}} U_{L_{i}}, \quad 1 \leqslant i \leqslant s,
$$

be the finite set of standard parabolic subgroups of $G$. We say that $\delta^{\prime} \in\left(\hat{L}_{j}\right)_{\mathrm{lds}}$ is affiliated to $\delta \in\left(\hat{L}_{i}\right)_{\mathrm{lds}}$, if $P_{L_{i}} \subset P_{L_{j}}$ and $\delta^{\prime}$ is a subrepresentation of $\operatorname{ind}_{P_{L_{i}}^{L_{j}} L_{j}^{1}}^{1}\left(\delta \otimes e^{0}\right)$ (cf. [CD90, Définition 2]). Given $L^{\prime} \in \mathcal{L}\left(t^{\prime}\right), L \in \mathcal{L}(t)$, and $\delta^{\prime} \in\left(\hat{L}^{\prime}\right)_{\text {lds }, t^{\prime}}$ affiliated to $\delta \in(\hat{L})_{\text {lds }, t}$, we have the intertwining operators $T\left(\delta^{\prime}\right)$ and $T(\delta)$ of $\S 4$ which intertwine $\operatorname{ind}_{P_{L^{\prime}}}^{G}\left(\delta \otimes e^{0}\right)$ and $\operatorname{ind}_{P_{L}}^{G}\left(\delta \otimes e^{0}\right)$ with their respective

$\sigma_{0^{-}}$-conjugates. It is immediate that the space of $\operatorname{ind}_{P_{L^{\prime}}}^{G}\left(\delta^{\prime} \otimes e^{0}\right)$ is a subspace of $\operatorname{ind}_{P_{L}}^{G}\left(\delta \otimes e^{0}\right)$, so the restriction of $T(\delta)$ to this subspace is also an intertwining operator between $\operatorname{ind}_{P_{L^{\prime}}}^{G}\left(\delta^{\prime} \otimes e^{0}\right)$ and its $\sigma_{0}$-conjugate. This restricted operator might be different from $T\left(\delta^{\prime}\right)$, and this is the type of incompatibility we would like to rule out in our subsequent definitions.

The second property we wish our intertwining operators to satisfy is that they intertwine $\sigma_{0}$-stable representations in a manner that is invariant under conjugation by permutation matrices. To attain this property we must define two types of intertwining operators. The reason for this is illustrated by the following example.

Recall the notation of $\S 5$ and consider $G=\mathrm{SL}(4, \mathbb{R})$ and the representation $\delta^{ \pm}=(\mathbf{1}, \mathbf{1}, \operatorname{sgn}, \operatorname{sgn})$ of $M_{0}^{ \pm}$in which 1 denotes the trivial representation of $\mathrm{SL}^{ \pm}(1, \mathbb{R})$. The Levi subgroup $M_{0}$ belongs to $\mathcal{L}(0)$ and $\delta$ belongs to $\left(\hat{M}_{0}\right)_{\mathrm{lds}, 0}$. It is clear that $R_{\delta}$ is non-trivial and that $w_{0}$ is a representative 


\section{P. MEZO}

of the longest element in $Z_{W_{\delta}}\left(R_{\delta}\right)$. According to Proposition $1, T(\delta)$ is an intertwining operator between $\operatorname{ind}_{P_{M_{0}}}^{G}\left(\delta \otimes e^{\nu}\right)$ and its $\sigma_{0}$-conjugate for all $\nu \in\left(\mathfrak{a}_{M_{0}, \mathbb{C}}^{w_{0}}\right)^{*}$. The operator $T(\delta)$ might appear to be all we need, but it does not intertwine some other $\sigma_{0}$-stable representations attached to $\delta$. Specifically, suppose $\nu$ is given by the 4 -tuple $\left(\nu_{1},-\nu_{1}, \nu_{2},-\nu_{2}\right)$ via the obvious embedding of $\mathfrak{a}_{M_{0}, \mathbb{C}}^{*}$ into $\mathbb{C}^{4}$. Suppose further that $\nu_{1}$ and $\nu_{2}$ are non-zero imaginary numbers and $\nu_{1} \neq \pm \nu_{2}$. Then one can show that $\operatorname{ind}_{P_{M_{0}}}^{G}\left(\delta \otimes e^{\nu}\right)$ is a $\sigma_{0}$-stable irreducible tempered representation. However,

$$
-w_{0} \nu=\left(\nu_{2},-\nu_{2}, \nu_{1},-\nu_{1}\right) \neq \nu
$$

so $\nu \notin\left(\mathfrak{a}_{M_{0}, \mathbb{C}}^{w_{0}}\right)^{*}$ and $T(\delta)$ does not intertwine ind $P_{M_{M_{0}}}^{G}\left(\delta \otimes e^{\nu}\right)$ with its $\sigma_{0}$-conjugate.

To find a different operator which does intertwine these representations, we can associate $\delta$ to the representation $\delta^{\prime}=(\mathbf{1}, \operatorname{sgn}, \operatorname{sgn}, \mathbf{1}) \in\left(\hat{M}_{0}\right)_{\mathrm{lds}, 0}$ by a permutation as in $\S 5$. Observe that in this case $w_{0}$ is a representative for the longest element in $W_{\delta^{\prime}}^{0}$, not $Z_{W_{\delta^{\prime}}}\left(R_{\delta^{\prime}}\right)$. We can then conjugate $T\left(\delta^{\prime}\right)$ by an operator of the form (8) to obtain the desired equivalence between $\operatorname{ind}_{P_{M_{0}}}^{G}\left(\delta \otimes e^{\nu}\right)$ and its $\sigma_{0}$-conjugate. We emphasize that the above two intertwining operators, $T(\delta)$ and $T\left(\delta^{\prime}\right)$, are distinguished by the two distinct types of Weyl groups, $W_{\delta}^{0}$ and $Z_{W_{\delta^{\prime}}}\left(R_{\delta^{\prime}}\right)$.

We shall begin our definitions by considering $Z_{W_{\delta}}\left(R_{\delta}\right)$ and defining an intertwining operator $T_{1}^{\sigma}(\delta, \nu)$ for $\delta \in\left(\hat{L}_{i}\right)_{\mathrm{lds}}, 1 \leqslant i \leqslant s$, and $\nu$ in a dense open subset of $\mathfrak{a}_{L_{i},}^{*}$. Suppose that $\delta \in\left(\hat{L}_{i}\right)_{\mathrm{lds}}$ lies in the discrete series. Define $\{\delta\}$ to be the equivalence class of all discrete series representations $\delta^{\prime} \in\left(\hat{L}_{j}\right)_{\text {lds }}$ such that $\delta=w \delta^{\prime}$ for some $w \in K$. If $\delta^{\prime} \in\left(\hat{L}_{j}\right)_{\text {lds }}$ belongs to $\{\delta\}$ then the set of minimal $K$ types (cf. [Kna86, ch. XV] ) of $\operatorname{ind}_{P_{L_{j}}}^{G}\left(\delta^{\prime} \otimes e^{0}\right)$ is equal to the set of minimal $K$ types of $\operatorname{ind}_{P_{L_{i}}}^{G}\left(\delta \otimes e^{0}\right)$. On the other hand, if two such representations belong to two different equivalence classes then their corresponding sets of minimal $K$ types are disjoint [CD84, Proposition 2]. This means that to each such equivalence class $\{\delta\}$ we can attach a minimal $K$ type $\mu_{\{\delta\}}$ which determines it.

Suppose $\delta \in\left(\hat{L}_{i}\right)_{\text {lds }}$ lies in the discrete series and $L_{i}$ belongs to $\mathcal{L}(t)$ for some $0 \leqslant t \leqslant\lfloor n / 2\rfloor$. The restriction of the operator $T(\delta)$ of (7) to the minimal $K$ type $\mu_{\{\delta\}}$ is a self-intertwining operator, as $\sigma_{0}$ fixes $K$ pointwise and $\mu_{\{\delta\}}$ is a $K$ type of multiplicity one in $\operatorname{ind}_{P_{L_{i}}}^{G}\left(\delta \otimes e^{0}\right)[\mathrm{Kna} 86$, ch. XV, $\S 1$, equation (1)]. This restriction is therefore given by a non-zero scalar $a_{\delta}$. Define

$$
T_{1}^{\sigma_{0}}(\delta, \nu)=a_{\delta}^{-1} T(\delta), \quad \nu \in\left(\mathfrak{a}_{L_{i}, \mathbb{C}}^{w_{t}}\right)^{*} .
$$

The operator $T_{1}^{\sigma_{0}}(\delta, \nu)$ intertwines $\operatorname{ind}_{P_{L_{i}}}^{G}\left(\delta \otimes e^{\nu}\right)$ with its $\sigma_{0}$-conjugate for any $\nu \in\left(\mathfrak{a}_{L_{i}, \mathbb{C}}^{w_{t}}\right)^{*}$, and its restriction to $\mu_{\{\delta\}}$ is the identity operator.

Suppose now that $\delta \in\left(\hat{L}_{i}\right)_{\text {lds }}$ lies in the discrete series, where $1 \leqslant i \leqslant s$ is arbitrary. Then $\delta$ satisfies the properties of Lemma 1 with $M=G, L=L_{i}$, and $\nu=0$. Therefore, by the arguments of $\S 5$, there is an integer $0 \leqslant t \leqslant\lfloor n / 2\rfloor$ and a permutation matrix $w \in G$ such that $w L_{i} w^{-1} \in \mathcal{L}(t)$, $w \delta \in\left(\widehat{w L_{i} w^{-1}}\right)_{\mathrm{lds}, t}$, and $w_{t}$ is the longest element in $Z_{W_{w \delta}}\left(R_{w \delta}\right)$. One can then compute that $w^{-1} w_{t} w$ is the longest element of $Z_{W_{\delta}}\left(R_{\delta}\right)$ with respect to the positive roots determined by $w^{-1} P_{w L_{i} w^{-1}} w$. As the Weyl group acts simply transitively on the Weyl chambers, there exists a unique element $w^{\prime \prime}$ in $Z_{W_{\delta}}\left(R_{\delta}\right)$ such that $\left(w w^{\prime \prime}\right)^{-1} w_{t} w w^{\prime \prime}$ is the longest element of $Z_{W_{\delta}}\left(R_{\delta}\right)$ with respect to the positive roots determined by $P_{L_{i}}$. We may therefore assume that $w^{-1} w_{t} w$ is the longest element of $Z_{W_{\delta}}\left(R_{\delta}\right)$ with respect to the positive basis determined by $P_{L_{i}}$.

We know that there exists an invertible operator $S(w, \delta, \nu)$ intertwining $\operatorname{ind}_{P_{L_{i}}}^{G}\left(\delta \otimes e^{\nu}\right)$ with $\operatorname{ind}_{P_{w L_{i} w^{-1}}}^{G}\left(w \delta \otimes e^{w \nu}\right)$ for $\nu$ in a dense open subset of $\mathfrak{a}_{L_{i}, \mathbb{C}}^{*}(\mathrm{cf}$. (8)). In fact $\nu \mapsto S(w, \delta, \nu)$ is a meromorphic map on $\mathfrak{a}_{L_{i}, \mathbb{C}}^{*}$. Define $T_{1}^{\sigma_{0}}(\delta, \nu)$ to be $S(w, \delta, \nu)^{-1} T(w \delta, w \nu) S(w, \delta, \nu)$. Clearly, $T_{1}^{\sigma_{0}}(\delta, \nu)$ intertwines $\operatorname{ind}_{P_{L_{i}}}^{G}\left(\delta \otimes e^{\nu}\right)$ with $\left(\operatorname{ind}_{P_{L_{i}}}^{G}\left(\delta \otimes e^{-w^{-1} w_{t} w \nu}\right)\right)^{\sigma_{0}}$ whenever $S(w, \delta, \nu)$ is defined, and its restriction to $\mu_{\{\delta\}}$ is the identity operator. 


\section{Twisted trace Paley-Wiener theorems}

Lemma 3. Suppose $\delta \in\left(\hat{L}_{i}\right)_{\mathrm{lds}}$ lies in the discrete series for some $1 \leqslant i \leqslant s$. Then $T_{1}^{\sigma_{0}}(\delta, \nu)$ is well-defined on a dense open subset of $\mathfrak{a}_{L_{i}, \mathbb{C}}^{*}$.

Proof. Suppose $w^{\prime}$ is another element of $G$ such that $w^{\prime} L_{i}\left(w^{\prime}\right)^{-1} \in \mathcal{L}\left(t^{\prime}\right), w^{\prime} \delta \in\left(w^{\prime} \widehat{L_{i}\left(w^{\prime}\right)^{-1}}\right)_{\mathrm{lds}, t^{\prime}}$, $w_{t^{\prime}}$ is the longest element in $Z_{W_{w^{\prime} \delta}}\left(R_{w^{\prime} \delta}\right)$, and $\left(w^{\prime}\right)^{-1} w_{t^{\prime}} w^{\prime}$ is the longest element in $Z_{W_{\delta}}\left(R_{\delta}\right)$. It is immediate that $\left(w^{\prime}\right)^{-1} w_{t^{\prime}} w^{\prime}=w^{-1} w_{t} w$ for $w$ as above, and so the operator

$$
S\left(w^{\prime}, \delta, \nu\right)^{-1} T\left(w^{\prime} \delta, w^{\prime} \nu\right) S\left(w^{\prime}, \delta, \nu\right)
$$

intertwines $\operatorname{ind}_{P_{L_{i}}}^{G}\left(\delta \otimes e^{\nu}\right)$ with $\left(\operatorname{ind}_{P_{L_{i}}}^{G}\left(\delta \otimes e^{-w^{-1} w_{t^{\prime}} w \nu}\right)\right)^{\sigma_{0}}$ for $\nu$ in a dense open subset of $\mathfrak{a}_{L_{i}, \mathbb{C}}^{*}$. According to [SV80], ind ${ }_{P_{L_{i}}}^{G}\left(\delta \otimes e^{\nu}\right)$ is irreducible for $\nu$ in an open subset of $\mathfrak{a}_{L_{i}, \mathbb{C}}^{*}$. Schur's lemma therefore implies that, for $\nu$ in an open subset of $\mathfrak{a}_{L_{i}, \mathbb{C}}^{*}, T_{1}^{\sigma_{0}}(\delta, \nu)$ is a scalar multiple of $S\left(w^{\prime}, \delta, \nu\right)^{-1}$ $T\left(w^{\prime} \delta, w^{\prime} \nu\right) S\left(w^{\prime}, \delta, \nu\right)$. The traces of the restrictions of these two operators to $\mu_{\{\delta\}}$ are equal (to the degree of $\left.\mu_{\{\delta\}}\right)$. Therefore, the scalar multiple must be 1 and $T_{1}^{\sigma_{0}}(\delta, \nu)$ equals $S\left(w^{\prime}, \delta, \nu\right)^{-1} T\left(w^{\prime} \delta\right)$ $S\left(w^{\prime}, \delta, \nu\right)$ for $\nu$ in an open subset of $\mathfrak{a}_{L_{i}, \mathbb{C}}^{*}$. By analytic continuation the equality holds for all $\nu \in \mathfrak{a}_{L_{i}, \mathbb{C}}^{*}$.

Suppose now that $1 \leqslant j \leqslant s$ and $\delta^{\prime} \in\left(\hat{L}_{j}\right)_{\text {lds }}$ is a non-degenerate limit of discrete series. It is affiliated to some $\delta \in\left(\hat{L}_{i}\right)_{\text {lds }}$ lying in the discrete series for some $1 \leqslant i \leqslant s$ [Kna86, Theorem 14.71]). The operator $T_{1}^{\sigma_{0}}(\delta, \nu)$ is defined for $\nu$ in the positive Weyl chamber of $\mathfrak{a}_{L_{j}, \mathbb{C}}^{*}$ (cf. the proof of Lemma 14.1 in [Kna86]). Since $T_{1}^{\sigma_{0}}(\delta, \nu)$ is meromorphic in $\nu$, it extends to a meromorphic function on all of $\mathfrak{a}_{L_{j}, \mathbb{C}}^{*}$. We define $T_{1}^{\sigma_{0}}\left(\delta^{\prime}, \nu\right)$ to be the restriction of $T_{1}^{\sigma_{0}}(\delta, \nu)$ to $\operatorname{ind}_{P_{L_{j}}}^{G}\left(\delta^{\prime} \otimes e^{\nu}\right)$ for $\nu$ in the dense open subset of $\mathfrak{a}_{L_{j}, \mathbb{C}}^{*}$ upon which $T_{1}^{\sigma_{0}}\left(\delta^{\prime}, \nu\right)$ is defined.

Lemma 4. Suppose $1 \leqslant j \leqslant s$ and $\delta^{\prime} \in\left(\hat{L}_{j}\right)_{\mathrm{lds}}$ is a non-degenerate limit of discrete series. Then $T_{1}^{\sigma_{0}}\left(\delta^{\prime}, \nu\right)$ is well-defined on a dense open subset of $\mathfrak{a}_{L_{j}, \mathbb{C}}^{*}$.

Proof. Suppose $\delta^{\prime}$ is affiliated to another representation $\delta^{\prime \prime} \in\left(\hat{L}_{i^{\prime}}\right)_{\text {lds }}$ lying in the discrete series for some $1 \leqslant i^{\prime} \leqslant s$. Then, by the Langlands disjointness theorem [Kna86, Theorem 14.90], there exists $w \in K \cap L_{j}$ such that $w \delta^{\prime \prime}=\delta$. We have the intertwining operator

$$
S=\mathcal{A}\left(P_{L_{i}} \cap L_{j}^{1}: w P_{L_{i^{\prime}}} w^{-1} \cap L_{j}^{1}: \delta: 0\right) \mathrm{L}(w)
$$

from ind ${ }_{P_{L_{i^{\prime}}} \cap L_{j}^{1}}^{L_{j}^{1}}\left(\delta^{\prime \prime} \otimes e^{0}\right)$ to ind ${ }_{P_{L_{i}} \cap L_{j}^{1}}^{L_{j}^{1}}\left(\delta \otimes e^{0}\right)$. The operator $S$ also induces an intertwining operator from $\operatorname{ind}_{P_{L_{i^{\prime}}}}^{G}\left(\delta^{\prime \prime} \otimes e^{\nu}\right)$ to $\operatorname{ind}_{P_{L_{i}}}^{G}\left(\delta \otimes e^{\nu}\right)$, by virtue of the equivalence

$$
\operatorname{ind}_{P_{L_{j}}}^{G}\left(\operatorname{ind}_{P_{L_{i^{\prime}}} \cap L_{j}}^{L_{j}}\left(\delta^{\prime \prime}\right) \otimes e^{\nu}\right) \cong \operatorname{ind}_{P_{L_{i^{\prime}}}}^{G}\left(\delta^{\prime \prime} \otimes e^{\nu}\right) .
$$

We also denote this induced operator by $S$. Since $\delta^{\prime}$ occurs in $\operatorname{ind}_{P_{L_{i}} \cap L_{j}^{1}}^{L^{1}}\left(\delta \otimes e^{0}\right)$ with multiplicity one [Kna86, Corollary 14.66] and $S^{-1} \delta^{\prime} S=\delta^{\prime}$, the restriction of $S^{-1} T_{1}^{\sigma 0}(\delta, \nu) S$ to ind $\operatorname{P}_{L_{j}}^{G}\left(\delta^{\prime} \otimes e^{\nu}\right)$ is equal to the restriction of $T_{1}^{\sigma_{0}}(\delta, \nu)$ to the same space. Obviously, $S^{-1} T_{1}^{\sigma_{0}}\left(\delta, \nu^{\prime}\right) S$ and $T_{1}^{\sigma_{0}}\left(\delta^{\prime \prime}, \nu^{\prime}\right)$ both intertwine ind $\operatorname{P}_{L_{i^{\prime}}}^{G}\left(\delta^{\prime \prime} \otimes e^{\nu^{\prime}}\right)$ with $\left(\operatorname{ind}_{P_{L_{i^{\prime}}}}^{G}\left(\delta^{\prime \prime} \otimes e^{-w^{\prime \prime} \nu^{\prime}}\right)\right)^{\sigma_{0}}$ for $w^{\prime \prime}$, the longest element in $Z_{W_{\delta}}\left(R_{\delta}\right)$, and $\nu^{\prime}$ in an open subset of $\mathfrak{a}_{L_{i^{\prime}}, \mathbb{C}}^{*}$.

We shall finish the proof by showing that $S^{-1} T_{1}^{\sigma_{0}}\left(\delta, \nu^{\prime}\right) S$ is equal to $T_{1}^{\sigma_{0}}\left(\delta^{\prime \prime}, \nu^{\prime}\right)$. By [SV80], $\operatorname{ind}_{P_{L_{i^{\prime}}}}^{G}\left(\delta^{\prime \prime} \otimes e^{\nu^{\prime}}\right)$ is irreducible for $\nu^{\prime}$ in an open subset of $\mathfrak{a}_{L_{i^{\prime}}, \mathbb{C}}^{*}$. This implies that $S^{-1} T_{1}^{\sigma 0}\left(\delta, \nu^{\prime}\right) S$ is a scalar multiple of $T_{1}^{\sigma_{0}}\left(\delta^{\prime \prime}, \nu^{\prime}\right)$. The traces of the restrictions of these operators to $\mu_{\{\delta\}}$ are equal. In consequence the scalar multiple must be one and the operators are equal.

Now that we have defined $T_{1}^{\sigma_{0}}\left(\delta^{\prime}, \nu\right)$ we ought to ensure that it is an operator intertwining the desired representations. 


\section{P. MEZO}

Lemma 5. Suppose $1 \leqslant j \leqslant s, \delta^{\prime} \in\left(\hat{L}_{j}\right)_{\mathrm{lds}}$ is a non-degenerate limit of discrete series, and $w$ is the longest element of $Z_{W_{\delta^{\prime}}}\left(R_{\delta^{\prime}}\right)$. Then $T_{1}^{\sigma_{0}}\left(\delta^{\prime}, \nu\right)$ intertwines $\operatorname{ind}_{P_{L_{j}}}^{G}\left(\delta^{\prime} \otimes e^{\nu}\right)$ with its $\sigma_{0}$-conjugate for $\nu$ in a dense open subset of $\left(\mathfrak{a}_{L_{i}, \mathbb{C}}^{w}\right)^{*}$.

Proof. We suppose first that $L_{j} \in \mathcal{L}(t)$ and $w=w_{t}$ is the longest element in $Z_{W_{\delta^{\prime}}}\left(R_{\delta^{\prime}}\right)$ for some $0 \leqslant t \leqslant\lfloor n / 2\rfloor$. Suppose also that $\delta^{\prime}$ is affiliated to a discrete series representation $\delta \in\left(\hat{L}_{i}\right)_{\mathrm{lds}}$ as above, $\nu \in\left(\mathfrak{a}_{L_{j}, \mathbb{C}}^{w_{t}}\right)^{*}$, and that $\left(\delta^{\prime}\right)^{ \pm}$is a representation of $L_{j}^{ \pm}$as in $\S 5$. In this case, $T_{1}^{\sigma_{0}}(\delta, \nu)$ is defined.

Since the only limit of discrete series representations occurring in the expansion of $\left(\delta^{\prime}\right)^{ \pm}$are induced from a Borel subgroup $B$ and are of the form

$$
\operatorname{ind}_{B}^{\mathrm{SL}^{ \pm}(2, \mathbb{R})}\left((\mathbf{1} \otimes \operatorname{sgn}) \otimes e^{0}\right)=\operatorname{ind}_{B}^{\mathrm{SL}^{ \pm}(2, \mathbb{R})}\left((\operatorname{sgn} \otimes \mathbf{1}) \otimes e^{0}\right)
$$

(cf. [Kna79, § 2]), we know that the expansion of $\delta^{ \pm}$is given by replacing some of these representations in $\left(\delta^{\prime}\right)^{ \pm}$with $(\mathbf{1}, \operatorname{sgn})$ or $(\operatorname{sgn}, \mathbf{1})$. A simple reordering of the latter representations implies the existence of $w^{\prime} \in L_{j}$, which normalizes $L_{i}$ and satisfies $w^{\prime} \delta \in\left(\hat{L}_{i}\right)_{\mathrm{lds}, t}$ with $w_{t}$ as the longest element in $Z_{W_{\delta}}\left(R_{\delta}\right)$. In addition, since

$$
T_{1}^{\sigma_{0}}(\delta, \nu)=T_{1}^{\sigma_{0}}\left(w^{\prime} \delta, w^{\prime} \nu\right)=T_{1}^{\sigma_{0}}\left(w^{\prime} \delta, \nu\right),
$$

we may assume that $w^{\prime}=1$. Thus, the restriction of $T_{1}^{\sigma_{0}}(\delta, \nu)$ to $\operatorname{ind}_{P_{L_{j}}}^{G}\left(\delta^{\prime} \otimes e^{\nu}\right)$ intertwines this representation with its $\sigma_{0}$-conjugate.

Dropping the assumption that $L_{j} \in \mathcal{L}(t)$, the normalization of [Kna86, Lemma 14.1] still ensures that $T_{1}\left(\delta^{\prime}, \nu\right)$ is defined for $\nu$ in a dense open subset (the positive Weyl chamber) of $\left(\mathfrak{a}_{L_{j}, \mathbb{C}}^{w}\right)^{*}$. We may therefore use the previous arguments to prove the lemma in this case as well.

Thus far we have defined an operator $T_{1}(\delta, \nu)$ which intertwines $\operatorname{ind}_{P_{L_{i}}}^{G}\left(\delta \otimes e^{\nu}\right)$ with its $\sigma_{0}$-conjugate for every $\delta \in\left(\hat{L}_{i}\right)_{\mathrm{lds}}, 1 \leqslant i \leqslant s$, and $\nu$ in a dense open subset of $\left(\mathfrak{a}_{L_{i}, \mathbb{C}}^{w}\right)^{*}$, where $w$ is the longest element in $Z_{W_{\delta}}\left(R_{\delta}\right)$. It is apparent from the definitions that these intertwining operators are compatible with respect to affiliation.

The example at the beginning of this section illustrates the need for an operator $T_{2}^{\sigma_{0}}(\delta, \nu)$ for $\delta \in\left(\hat{L}_{i}\right)_{\operatorname{lds}}, 1 \leqslant i \leqslant s$, and $\nu$ in a dense open subset of $\left(\mathfrak{a}_{L_{i}, \mathbb{C}}^{w}\right)^{*}$ where $w$ is now the longest element in $W_{\delta}^{0}$. We define this second type of intertwining operator by following the definition of $T_{1}^{\sigma_{0}}(\delta, \nu)$ and replacing $Z_{W_{\delta}}\left(R_{\delta}\right)$ everywhere with $W_{\delta}^{0}$.

It is important to realize that if $n$ is odd then $T_{1}^{\sigma_{0}}(\delta, \nu)$ is equal to $T_{2}^{\sigma_{0}}(\delta, \nu)$. Indeed, we know from $\S 5$ that the only way the $\mathrm{R}$ group of $\delta$ can be non-trivial is if the trivial character and sign character appear in $\delta^{ \pm}$an equal number of times. This is only possible if $n$ is even.

\section{7. $\sigma_{0}$-twisted trace Paley-Wiener theorems}

Our strategy in proving a $\sigma_{0}$-twisted trace Paley-Wiener theorem for $\operatorname{SL}(n, \mathbb{R})$ is to follow [CD84] and [CD90]. We first prove an analogue of their Proposition 1 [CD84], which deals with individual representations in $(\hat{L})_{\mathrm{lds}, t}$ for some $L \in \mathcal{L}(t)$ and $0 \leqslant t \leqslant\lfloor n / 2\rfloor$. Then we prove a $\sigma_{0}$-twisted analogue of their trace Paley-Wiener theorems for two different cases, depending on some $\mathrm{R}$ groups.

To refer to the proof of Proposition 1 [CD84] we need some notation. Let $N$ be a positive real number. Suppose $L=L_{i}$ for some $1 \leqslant i \leqslant s$ and define $\mathcal{P} \mathcal{W}\left(\mathfrak{a}_{L}\right)_{N}$ to be the image under the Fourier transform of the smooth functions on $\mathfrak{a}_{L}$ with support in the closed ball of radius $N$ about the origin. The classical Paley-Wiener theorem tells us that functions $\phi$ in $\mathcal{P} \mathcal{W}\left(\mathfrak{a}_{L}\right)_{N}$ are entire on 


\section{Twisted trace Paley-Wiener theorems}

$\mathfrak{a}_{L, \mathbb{C}}^{*}$ and satisfy a growth condition,

$$
\sup _{\lambda \in \mathfrak{a}_{L, \mathbb{C}}^{*}}\left\{|\phi(\lambda)| e^{-N|\operatorname{Re}(\lambda)|}(1+|\operatorname{Im}(\lambda)|)^{k} t\right\}<\infty,
$$

for every integer $k$. If $W$ is a group acting on $\mathfrak{a}_{L}$ we denote the $W$-invariant subspace of $\mathcal{P} \mathcal{W}\left(\mathfrak{a}_{L}\right)_{N}$ by $\mathcal{P} \mathcal{W}\left(\mathfrak{a}_{L}\right)_{N}^{W}$.

Suppose now that $L \in \mathcal{L}(t), \mathfrak{k}$ is the Lie algebra of $K$ and $\mathfrak{t} \subset \mathfrak{k}$ is the Lie algebra of a compact Cartan subgroup of $L^{1}$. Then

$$
\mathfrak{h}=\mathfrak{t} \oplus \mathfrak{a}_{L}=\mathfrak{t} \oplus \mathfrak{a}_{L}^{w_{t}} \oplus\left(\mathfrak{a}_{L}^{w_{t}}\right)^{\perp}
$$

is a Cartan subalgebra of $\mathfrak{g}$, the Lie algebra of $G$. Let $W(\mathfrak{h})$ be the Weyl group of $\mathfrak{h}, S(\mathfrak{h})$ be the polynomial algebra on $\mathfrak{h}_{\mathbb{C}}^{*}$, and $\mathcal{P} \mathcal{W}(\mathfrak{h})$ be the Paley-Wiener space determined by $\mathfrak{h}$. As before, we denote the $W$-invariant subspaces of $S(\mathfrak{h})$ and $\mathcal{P} \mathcal{W}(\mathfrak{h})$ by $S(\mathfrak{h})^{W}$ and $\mathcal{P W}(\mathfrak{h})^{W}$, respectively, for any group $W$ acting on $\mathfrak{h}$.

Define $C_{c}^{\infty}(G, K)_{N}$ to be the space of smooth $K$-finite functions of $G$ with support in $K \exp (\mathfrak{a}(N)) K$, where $\mathfrak{a}(N)$ is the closed ball of radius $N$ about the origin in $\mathfrak{a}_{M_{0}}$.

Proposition 3. Suppose $k=1,2,0 \leqslant t \leqslant\lfloor n / 2\rfloor, L \in \mathcal{L}(t), \delta \in(\hat{L})_{\text {lds }, t}, \mu$ is a minimal $K$ type of $\operatorname{ind}_{P_{L}}^{G}\left(\delta \otimes e^{0}\right)$, and $F$ is a complex function of $\left(\mathfrak{a}_{L, \mathbb{C}}^{w_{t}}\right)^{*}$. Suppose further that

$$
W=\left\{w \in W_{\delta}: w \mathfrak{a}_{L}^{w_{t}} \subset \mathfrak{a}_{L}^{w_{t}}\right\} .
$$

Then there exists $f \in C_{c}^{\infty}(G, K)_{N}$ of type $(\mu, \mu)$ such that

$$
F(\nu)=\operatorname{tr}\left(\operatorname{ind}_{P_{L}}^{G}\left(\delta \otimes e^{\nu}\right)(f) T_{k}^{\sigma_{0}}(\delta, \nu)\right)
$$

if and only if $F$ belongs to $\mathcal{P} \mathcal{W}\left(\mathfrak{a}_{L}^{w_{t}}\right)_{N}^{W}$.

Proof. Suppose $F \in \mathcal{P} \mathcal{W}\left(\mathfrak{a}_{L}^{w_{t}}\right)_{N}^{W}$. By the Corollary of [Cow86], $F$ extends to a function $F^{\prime} \in$ $\mathcal{P W}(\mathfrak{h})_{N}^{W}$ such that

$$
F^{\prime}\left(\lambda_{\delta}+\nu\right)=F(\nu), \quad \nu \in\left(\mathfrak{a}_{L, \mathbb{C}}^{w_{t}}\right)^{*}
$$

for the Harish-Chandra parameter $\lambda_{\delta} \in \mathfrak{t}_{\mathbb{C}}^{*}$ of $\delta$ (cf. [Kna86, $\S 7$, ch. IX]). By a theorem of Raïs (cf. [CD84, Lemme 8]), it follows that

$$
S(\mathfrak{h})^{W} \mathcal{P} \mathcal{W}(\mathfrak{h})_{N}^{W(\mathfrak{h})}=\mathcal{P} \mathcal{W}^{W}(\mathfrak{h})_{N} .
$$

We therefore write $F^{\prime}(\Lambda)=\sum P_{i}(\Lambda) F_{i}^{\prime}(\Lambda)$, where $P_{i} \in S(\mathfrak{h})^{W}$ and $F_{i}^{\prime} \in \mathcal{P} \mathcal{W}(\mathfrak{h})_{N}^{W(\mathfrak{h})}$. Since the involution given by

$$
\lambda \mapsto-w_{t} \lambda, \quad \lambda \in \mathfrak{a}_{L}^{*}
$$

stabilizes $\Delta_{\delta}^{0} \cap \Delta_{L}^{+},\left[\right.$CD90, Proposition A.1] tells us that there exists a function $P_{i}^{\prime \prime} \in S(\mathfrak{h})^{W_{\delta}^{0}}$ which agrees with $P_{i}$ on $\lambda_{\delta}+\left(\mathfrak{a}_{L, \mathbb{C}}^{w_{t}}\right)^{*}$. As $R_{\delta} \subset W$, the function

$$
P_{i}^{\prime}(\lambda)=\left|R_{\delta}\right|^{-1} \sum_{r \in R_{\delta}} P_{i}^{\prime \prime}(r \Lambda), \quad \Lambda \in \mathfrak{h}_{\mathbb{C}}^{*}
$$

is a function in $S(\mathfrak{h})^{W_{\delta}}$ which agrees with $P_{i}$ on $\lambda_{\delta}+\left(\mathfrak{a}_{L, \mathbb{C}}^{w_{t}}\right)^{*}$. By [CD84, Theorem 2] there exists a $\mathfrak{k}$-invariant element $u_{i}$ in the universal enveloping algebra of $\mathfrak{g}$ which acts on the minimal $K$ type $\mu$ of $\operatorname{ind}_{P_{L}}^{G}\left(\delta \otimes e^{\lambda}\right)$ as multiplication by

$$
P_{i}^{\prime}\left(\lambda_{\delta}+\lambda\right)=P_{i}\left(\lambda_{\delta}+\lambda\right), \quad \lambda \in \mathfrak{a}_{L, \mathbb{C}}^{*}
$$

By [CD84, Lemma 9] there exists $f_{i} \in C_{c}^{\infty}(G, K)_{N}$ of type $(\mu, \mu)$ such that, for all $\lambda \in\left(\mathfrak{a}_{L, \mathbb{C}}^{w_{t}}\right)^{*}$, $\operatorname{ind}_{P_{L}}^{G}\left(\delta \otimes e^{\lambda}\right)\left(f_{i}\right)$ equals $F_{i}^{\prime}\left(\lambda_{\delta}+\lambda\right)$ on $\mu$ and equals zero on any other $K$ type. The restriction of 
$T_{k}^{\sigma_{0}}(\delta, \nu)$ to $\mu$ is a self-intertwining operator and hence a scalar $\varepsilon_{k}= \pm 1$. The function

$$
f=\varepsilon_{k} \cdot \frac{\sum u_{i} * f_{i}}{\operatorname{deg}(\mu)}
$$

can be seen to satisfy the claim of the proposition.

Suppose now that the converse holds. Since $f$ is $K$-finite, one may choose a basis of $\operatorname{ind}_{P_{L_{i}}}^{G}\left(\delta \otimes e^{\nu}\right)$, with respect to its $K$ types, such that only finitely many matrix coefficients of $\operatorname{ind}_{P_{L_{i}}}^{G}\left(\delta \otimes e^{\nu}\right)(f)$ are non-zero. According to $[\mathrm{CD} 84, \S 2.1]$, each non-zero matrix coefficient belongs to $\mathcal{P} \mathcal{W}\left(\mathfrak{a}_{L}\right)_{N}$. As the operator $T_{k}^{\sigma_{0}}(\delta, \nu)$ sends the $K$-isotypical components of $\operatorname{ind}_{P_{L_{i}}}^{G}\left(\delta \otimes e^{\nu}\right)$ to themselves and does not depend on $\nu$, there are finitely many non-zero matrix coefficients of ind ${ }_{P_{L_{i}}}^{G}\left(\delta \otimes e^{\nu}\right)(f) T_{k}^{\sigma_{0}}(\delta, \nu)$, and each of these matrix coefficients is a finite linear combination of functions in $\mathcal{P} \mathcal{W}\left(\mathfrak{a}_{L}\right)_{N}$. Consequently, the trace of $\operatorname{ind}_{P_{L_{i}}}^{G}\left(\delta \otimes e^{\nu}\right)(f) T_{k}^{\sigma}(\delta, \nu)$ defines a function in $\mathcal{P} \mathcal{W}\left(\mathfrak{a}_{L}\right)_{N}$. The invariance of this function under $W$ is obvious.

Given $L \in \mathcal{L}(t)$, let $(\hat{L})_{\text {lds }, t}^{1}$ be the subset of representations $\delta \in(\hat{L})_{\text {lds }, t}$ such that $w_{t}$ is a representative of the longest element in $Z_{W_{\delta}}\left(R_{\delta}\right)$, and let $(\hat{L})_{\text {lds }, t}^{2}$ be the subset of representations $\delta \in(\hat{L})_{\text {lds }, t}$ such that $w_{t}$ is a representative of the longest element in $W_{\delta}^{0}$. The following theorem is a $\sigma_{0}$-twisted version of $[\mathrm{CD} 90$, Théorème 1] in the case $G=\mathrm{SL}(n, \mathbb{R})$.

Theorem 1. Suppose $N>0, k=1,2$, and that for each $0 \leqslant t \leqslant\lfloor n / 2\rfloor$ and $1 \leqslant i \leqslant s$ such that $L_{i} \in \mathcal{L}(t)$ we are given a function

$$
F_{i, t}^{k}:\left(\hat{L}_{i}\right)_{\mathrm{lds}, t}^{k} \times\left(\mathfrak{a}_{L_{i}, \mathbb{C}}^{w_{t}}\right)^{*} \rightarrow \mathbb{C} .
$$

Then the following are equivalent.

a) There exists $f_{k} \in C_{c}^{\infty}(G, K)_{N}$ such that

$$
F_{i, t}^{k}(\delta, \nu)=\operatorname{tr}\left(\operatorname{ind}_{P_{L_{i}}}^{G}\left(\delta \otimes e^{\nu}\right)\left(f_{k}\right) T_{k}^{\sigma_{0}}(\delta, \nu)\right), \quad \nu \in\left(\mathfrak{a}_{L_{i}, \mathbb{C}}^{w_{t}}\right)^{*} .
$$

b) (1) $F_{i, t}^{k}$ has finite support.

(2) $F_{i, t}^{k}(\delta, \cdot)$ belongs to $\mathcal{P} \mathcal{W}\left(\mathfrak{a}_{L_{i}}^{w_{t}}\right)_{N}$, for all $\delta \in\left(\hat{L}_{i}\right)_{\mathrm{lds}, t}^{k}$.

(3) Suppose $\delta \in\left(\hat{L}_{i}\right)_{\mathrm{lds}, t}^{k}, \nu \in\left(\mathfrak{a}_{L_{i}, \mathbb{C}}^{w_{t}}\right)^{*}$, and $w \in K$ such that $w \delta \in\left(\hat{L}_{j}\right)_{\mathrm{lds}, t}^{k}$ and $w \mathfrak{a}_{L_{i}}^{w_{t}}=\mathfrak{a}_{L_{j}}^{w_{t}}$. Then $F_{i, t}^{k}(\delta, \nu)=F_{j, t}^{k}(w \delta, w \nu)$.

(4) Suppose $1 \leqslant i, j \leqslant s, P_{L_{i}} \subset P_{L_{j}}, \delta \in\left(\hat{L}_{i}\right)_{\mathrm{lds}, t}^{k}, \delta_{1}^{\prime}, \ldots, \delta_{m}^{\prime} \in\left(\hat{L}_{j}\right)_{\mathrm{lds}, t}^{k}$, and

$$
\operatorname{ind}_{P_{L_{i}} \cap L_{j}^{1}}^{L_{j}^{1}}\left(\delta \otimes e^{0}\right)=\delta_{1}^{\prime} \oplus \cdots \oplus \delta_{m}^{\prime} .
$$

Then

$$
F_{i, t}^{k}(\delta, \nu)=F_{j, t}^{k}\left(\delta_{1}^{\prime}, \nu\right)+\cdots+F_{j, t}^{k}\left(\delta_{m}^{\prime}, \nu\right), \nu \in\left(\mathfrak{a}_{L_{j}, \mathbb{C}}^{w_{t}}\right)^{*} .
$$

Proof. We start the proof by assuming condition a holds and showing that each condition listed under item $\mathrm{b}$ is satisfied. In doing this, we follow [CD84, $\S 2.1]$. To prove item $\mathrm{b}(1)$ we may assume that $\mu_{1}, \mu_{2}$ are irreducible representations of $K$ and that $f_{k}$ is of type $\left(\mu_{1}, \mu_{2}\right)$. It can then be shown that if $\operatorname{ind}_{P_{L_{i}}}^{G}\left(\delta \otimes e^{\nu}\right)\left(f_{k}\right) \neq 0$ then $\mu_{1}=\mu_{2}$ and $\mu_{1}$ is a $K$ type of $\operatorname{ind}_{P_{L_{i}}}^{G}\left(\delta \otimes e^{\nu}\right)$. Frobenius reciprocity implies that the restriction of $\mu_{1}$ to $K \cap L_{i}$ contains a $\left(K \cap L_{i}\right)$-type of $\delta$. A result of Harish-Chandra tells us that there are only finitely many inequivalent irreducible admissible representations of $L_{i}$ containing a fixed $\left(K \cap L_{i}\right)$-type, from which b(1) follows.

Condition $b(2)$ holds as in the proof of Proposition 3. 


\section{Twisted trace Paley-Wiener theorems}

Suppose the hypothesis of $\mathrm{b}(3)$ holds. It is then clear from the definitions of $\S 6$ that $T_{k}^{\sigma_{0}}(\delta, \nu)$ is equal to $S(w, \delta, \nu)^{-1} T_{k}^{\sigma_{0}}(w \delta, w \nu) S(w, \delta, \nu)$. We therefore have

$$
\begin{aligned}
\operatorname{tr}\left(\operatorname{ind}_{P_{L_{i}}}^{G}\left(\delta \otimes e^{\nu}\right)\left(f_{k}\right) T_{k}^{\sigma_{0}}(\delta, \nu)\right) & =\operatorname{tr}\left(\operatorname{ind}_{P_{L_{i}}}^{G}\left(\delta \otimes e^{\nu}\right)\left(f_{k}\right) S(w, \delta, \nu)^{-1} T_{k}^{\sigma_{0}}(w \delta, w \nu) S(w, \delta, \nu)\right) \\
& =\operatorname{tr}\left(S(w, \delta, \nu) \operatorname{ind}_{P_{L_{i}}}^{G}\left(\delta \otimes e^{\nu}\right)\left(f_{k}\right) S(w, \delta, \nu)^{-1} T_{k}^{\sigma 0}(w \delta, w \nu)\right) \\
& =\operatorname{tr}\left(\operatorname{ind}_{P_{L_{j}}}^{G}\left(w \delta \otimes e^{w \nu}\right)\left(f_{k}\right) T_{k}^{\sigma 0}(w \delta, w \nu)\right) .
\end{aligned}
$$

Suppose the hypothesis of $\mathrm{b}(4)$ holds and that $\nu \in\left(\mathfrak{a}_{L_{j}, \mathbb{C}}^{w_{t}}\right)^{*}$. Then $\operatorname{ind}_{P_{L_{i}}}^{G}\left(\delta \otimes e^{\nu}\right)$ is equivalent to

$$
\operatorname{ind}_{P_{L_{j}}}^{G}\left(\delta_{1}^{\prime} \otimes e^{\nu}\right) \oplus \cdots \oplus \operatorname{ind}_{P_{L_{j}}}^{G}\left(\delta_{m}^{\prime} \otimes e^{\nu}\right) .
$$

By definition, the restriction of $T_{k}^{\sigma_{0}}(\delta, \nu)$ to $\operatorname{ind}_{P_{L_{j}}}^{G}\left(\delta_{b}^{\prime} \otimes e^{\nu}\right)$ is equal to $T_{k}^{\sigma_{0}}\left(\delta_{b}^{\prime}, \nu\right)$, for $1 \leqslant b \leqslant m$. Thus the trace of $\operatorname{ind}_{P_{L_{i}}}^{G}\left(\delta \otimes e^{\nu}\right)\left(f_{k}\right) T_{k}^{\sigma_{0}}(\delta, \nu)$ is equal to the trace of

$$
\operatorname{ind}_{P_{L_{j}}}^{G}\left(\delta_{1}^{\prime} \otimes e^{\nu}\right)\left(f_{k}\right) T_{k}^{\sigma_{0}}\left(\delta_{1}^{\prime}, \nu\right) \oplus \cdots \oplus \operatorname{ind}_{P_{L_{j}}}^{G}\left(\delta_{m}^{\prime} \otimes e^{\nu}\right)\left(f_{k}\right) T_{k}^{\sigma_{0}}\left(\delta_{m}^{\prime}, \nu\right),
$$

and the conclusion of $\mathrm{b}(4)$ ensues.

Having proved that (a) implies (b) we prove the converse. Suppose the conditions of (b) hold and that $\delta \in\left(\hat{L}_{i}\right)_{\text {lds }, t}^{k}$ belongs to the discrete series for some $L_{i} \in \mathcal{L}(t)$. According to Proposition 3 and Proposition 1 of [CD90], there exists a function $f^{\prime} \in C_{c}^{\infty}(G, K)_{N}$ such that

$$
\operatorname{tr}\left(\operatorname{ind}_{P_{L_{j}}}^{G}\left(\delta^{\prime} \otimes e^{\nu}\right)\left(f^{\prime}\right)\right)=F_{j, t}^{k}\left(\delta^{\prime}, \nu\right), \quad 1 \leqslant j \leqslant s,
$$

for all $\delta^{\prime} \in\left(\hat{L}_{j}\right)_{\mathrm{lds}, t}^{k}$ affiliated to $\delta$ and $\nu \in\left(\mathfrak{a}_{L_{j}, \mathbb{C}}^{w_{t}}\right)^{*}$. Before we move on, some justification of the use of [CD90, Proposition 1] is in order. Clozel and Delorme make use of a space $\mathcal{P} \mathcal{W}\left(E, W, \Delta^{+}\right)_{r}$, where $r>0, E$ is a real vector space, $\Delta^{+}$is a set of positive roots of a root system $\Delta$ of a subspace of $E$, and $W$ is a group of automorphisms of $E$ containing the Weyl group $W^{0}$ of $\Delta$ as a normal subgroup such that $W / W^{0}$ is isomorphic to a product of copies of $\mathbb{Z} / 2 \mathbb{Z}$ [CD90, Appendice C]. We apply the machinery of $\mathcal{P} \mathcal{W}\left(E, W, \Delta^{+}\right)_{r}$ to the present context by taking $r=N, E=\mathfrak{a}_{L_{i}}^{w_{t}}$ and

$$
\Delta=\Delta_{\delta}^{0} \cap\left(\mathfrak{a}_{L_{i}, \mathbb{C}}^{w_{t}}\right)^{*} .
$$

We need to take

$$
W=\left\{w \in W_{\delta}: w \mathfrak{a}_{L_{i}}^{w_{t}} \subset \mathfrak{a}_{L_{i}}^{w_{t}}\right\},
$$

despite the fact that $W / W^{0}$ is not necessarily isomorphic to a product of copies of $\mathbb{Z} / 2 \mathbb{Z}$. This choice for $W$ is legitimate if we replace $W^{0}$ with $W \cap W_{\delta}^{0}$ in the formalism of [CD90, Appendice C]. Indeed, $W /\left(W \cap W_{\delta}^{0}\right)$ is isomorphic to a product of copies of $\mathbb{Z} / 2 \mathbb{Z}$, and the remaining results of Appendice $\mathrm{C}$ also remain valid with this choice of $W^{0}$.

Moving on, the function $f^{\prime}$ is the sum of functions $f_{\mu} \in C_{c}^{\infty}(G, K)_{N}$, where $\mu$ is a minimal $K$ type of $\operatorname{ind}_{P_{L_{i}}}^{G}\left(\delta \otimes e^{0}\right)$ and $f_{\mu}$ is of type $(\mu, \mu)$. The restriction of the operator $T_{k}^{\sigma_{0}}(\delta, \nu)$ to $\mu$ is a self-intertwining operator and hence given by a scalar $\varepsilon_{k}(\delta, \mu)= \pm 1$. Setting $g_{k}$ to be the sum of the functions $\varepsilon_{k}(\delta, \mu) f_{\mu}$, where $\mu$ runs over the minimal $K$ types of $\operatorname{ind}_{P_{L_{i}}}^{G}\left(\delta \otimes e^{0}\right)$, we see that $g_{k} \in C_{c}^{\infty}(G, K)_{N}$ and

$$
\operatorname{tr}\left(\operatorname{ind}_{P_{L_{j}}}^{G}\left(\delta^{\prime} \otimes e^{\nu}\right)\left(g_{k}\right) T_{k}^{\sigma_{0}}\left(\delta^{\prime}, \nu\right)\right)=F_{j, t}^{k}\left(\delta^{\prime}, \nu\right), \quad 1 \leqslant j \leqslant s,
$$

for all $\delta^{\prime} \in\left(\hat{L}_{j}\right)_{\mathrm{lds}, t}$ affiliated to $\delta$ and $\nu \in\left(\mathfrak{a}_{L_{j}, \mathbb{C}}^{w_{t}}\right)^{*}$.

The existence of the function $g_{k}$ is a $\sigma_{0}$-twisted analogue of [CD90, Proposition 1]. It allows us to adapt the proofs of [CD90, Théorème 1] and [CD84, Théorème 1] to our context thereby completing the proof of this theorem. The cited proofs rely on an induction argument involving the support 


\section{P. MEZO}

of $F_{i, t}^{k}$ and the length of minimal $K$ types. The details of the induction argument can be found in $[\mathrm{CD} 84, \S 2.3]$.

The next corollary is proven by combining Theorem 1 with the definitions of $\S 6$ and equations like those of (10).

Corollary 1 . Given $\delta \in\left(\hat{L}_{i}\right)_{\mathrm{lds}}, 1 \leqslant i \leqslant s$, let $w_{\delta, 1}$ be the longest element of $Z_{W_{\delta}}\left(R_{\delta}\right)$ and $w_{\delta, 2}$ be the longest element of $W_{\delta}^{0}$. Suppose $N>0, k=1,2$, and that for each $1 \leqslant i \leqslant s$ we are given a function

$$
F_{i}^{k}:\left(\hat{L}_{i}\right)_{\mathrm{lds}} \times\left(\mathfrak{a}_{L_{i}, \mathbb{C}}^{w_{\delta, k}}\right)^{*} \rightarrow \mathbb{C}
$$

Then the following are equivalent.

a) There exists $f_{k} \in C_{c}^{\infty}(G, K)_{N}$ such that

$$
F_{i}^{k}(\delta, \nu)=\operatorname{tr}\left(\operatorname{ind}_{P_{L_{i}}}^{G}\left(\delta \otimes e^{\nu}\right)\left(f_{k}\right) T_{k}^{\sigma_{0}}(\delta, \nu)\right), \quad \nu \in\left(\mathfrak{a}_{L_{i}, \mathbb{C}}^{w_{\delta, k}}\right)^{*} .
$$

b) (1) $F_{i}^{k}$ has finite support.

(2) $F_{i}^{k}(\delta, \cdot)$ belongs to $\mathcal{P} \mathcal{W}\left(\mathfrak{a}_{L_{i}}^{w_{\delta, k}}\right)_{N}$, for all $\delta \in\left(\hat{L}_{i}\right)_{\mathrm{lds}}$.

(3) Suppose $\delta \in\left(\hat{L}_{i}\right)_{\mathrm{lds}}, \nu \in\left(\mathfrak{a}_{L_{i}, \mathbb{C}}^{w_{\delta, k}}\right)^{*}$, and $w \in K$ such that $w \delta \in\left(\hat{L}_{j}\right)_{\mathrm{lds}}$ and $w \mathfrak{a}_{L_{i}}^{w_{\delta, k}}=\mathfrak{a}_{L_{i}}^{w_{w, k}}$. Then $F_{i}^{k}(\delta, \nu)=F_{j}^{k}(w \delta, w \nu)$.

(4) Suppose $1 \leqslant i, j \leqslant s, P_{L_{i}} \subset P_{L_{j}}, \delta \in\left(\hat{L}_{i}\right)_{\mathrm{lds}}, \delta_{1}^{\prime}, \ldots, \delta_{m}^{\prime} \in\left(\hat{L}_{j}\right)_{\mathrm{lds}}$, and

$$
\operatorname{ind}_{P_{L_{i}} \cap L_{j}^{1}}^{L_{j}^{1}}\left(\delta \otimes e^{0}\right)=\delta_{1}^{\prime} \oplus \cdots \oplus \delta_{m}^{\prime} .
$$

Then

$$
F_{i}^{k}(\delta, \nu)=F_{j}^{k}\left(\delta_{1}^{\prime}, \nu\right)+\cdots+F_{j}^{k}\left(\delta_{m}^{\prime}, \nu\right), \quad \nu \in\left(\mathfrak{a}_{L_{j}, \mathbb{C}}^{w_{\delta, k}}\right)^{*}
$$

\section{A compatibility condition}

We mentioned at the end of $\S 6$ that if $n$ is odd then the $\mathrm{R}$ groups of our discussion are all trivial. In this case the parameter $k=1,2$ of Corollary 1 is superfluous. That is, a single function in $C_{c}^{\infty}(G, K)_{N}$ determines any set of functions satisfying the properties of Corollary 1(b).

Let us suppose for the rest of this section that $n$ is even and that we have functions $F_{j}^{k}$ for $1 \leqslant j \leqslant s$ and $k=1,2$, which satisfy the conditions of Theorem $1(\mathrm{~b})$. If $\left\{F_{j}^{1}\right\}$ and $\left\{F_{j}^{2}\right\}$ are compatible in some sense, then we should also be able to obtain a single function in $C_{c}^{\infty}(G, K)_{N}$ which satisfies an equality as in Corollary 1 (a) for both $k=1$ and $k=2$.

Let us make this notion of compatibility precise. Suppose $\delta \in\left(\hat{L}_{j}\right)_{\mathrm{lds}}$ has trivial R group. Then the Weyl group elements $w_{\delta, 1}$ and $w_{\delta, 2}$ of the corollary are equal and $\operatorname{ind}_{P_{L_{j}}}^{G}\left(\delta \otimes e^{\nu}\right)$ is irreducible for all $\nu \in i\left(\mathfrak{a}_{L_{j}}^{w_{\delta, 1}}\right)^{*}$. Under these circumstances $T_{1}^{\sigma_{0}}(\delta, \nu)$ and $T_{2}^{\sigma_{0}}(\delta, \nu)$ are defined [Kna86, Theorem 14.20(d)] and $T_{1}^{\sigma_{0}}(\delta, \nu) T_{2}^{\sigma_{0}}(\delta, \nu)$ is a self-intertwining operator of $\operatorname{ind}_{P_{L_{j}}}^{G}\left(\delta \otimes e^{\nu}\right)$. By Schur's lemma, it is given by a scalar $c(\delta, \nu) \in \mathbb{C}$. We say that $\left\{F_{j}^{1}\right\}$ is compatible with $\left\{F_{j}^{2}\right\}$ if

$$
F_{j}^{2}(\delta, \nu)=c(\delta, \nu) F_{j}^{1}(\delta, \nu), \quad \nu \in i\left(\mathfrak{a}_{L_{j}}^{w_{\delta, 1}}\right)^{*}
$$

for any $\delta$ as above. Such a compatibility condition is necessary, for if $\delta$ and $\nu$ are as above and $f \in C_{c}^{\infty}(G, K)_{N}$ then

$$
\begin{aligned}
\operatorname{tr}\left(\operatorname{ind}_{P_{L_{j}}}^{G}\left(\delta \otimes e^{\nu}\right)(f) T_{2}^{\sigma_{0}}(\delta, \nu)\right) & =\operatorname{tr}\left(\operatorname{ind}_{P_{L_{j}}}^{G}\left(\delta \otimes e^{\nu}\right)(f)\left(T_{1}^{\sigma_{0}}(\delta, \nu)\right)^{2} T_{2}^{\sigma_{0}}(\delta, \nu)\right) \\
& =c(\delta, \nu) \operatorname{tr}\left(\operatorname{ind}_{P_{L_{j}}}^{G}\left(\delta \otimes e^{\nu}\right)(f) T_{1}^{\sigma_{0}}(\delta, \nu)\right) .
\end{aligned}
$$




\section{Twisted trace Paley-Wiener theorems}

To prove the existence of the desired function in $C_{c}^{\infty}(G, K)_{N}$ from the compatibility condition, we wish to define a function on $\mathcal{P} \mathcal{W}\left(\mathfrak{a}_{L_{j}}\right)$ which restricts to $F_{j}^{k}(\delta, \cdot)$ on $i\left(\mathfrak{a}_{L_{j}}^{w_{\delta, k}}\right)^{*}$ for any $k=1,2$ and $\delta \in\left(\hat{L}_{j}\right)_{\mathrm{lds}}$. This can be accomplished using the following.

Conjecture $^{1} 1$. Suppose that $n$ is even, $N>0,1 \leqslant i \leqslant s, \delta \in\left(\hat{L}_{i}\right)_{\text {lds }}$ lies in the discrete series, $w_{\delta, 1}$ is the longest element of $Z_{W_{\delta}}\left(R_{\delta}\right)$, and $w_{\delta, 2}$ is the longest element of $W_{\delta}^{0}$. Suppose further that $\phi \in \mathcal{P} \mathcal{W}\left(\mathfrak{a}_{L_{i}}^{w_{\delta, 2}}\right)_{N}$ is invariant under

$$
\left\{w \in W_{\delta}: w \mathfrak{a}_{L_{i}}^{w_{\delta, 2}} \subset \mathfrak{a}_{L_{i}}^{w_{\delta, 2}}\right\}
$$

and vanishes on $\left(\mathfrak{a}_{L_{i}, \mathbb{C}}^{w_{\delta, 2}}\right)^{*} \cap\left(\mathfrak{a}_{L_{i}, \mathbb{C}}^{w_{\delta, 1}}\right)^{*}$. Then $\phi$ extends to a $W_{\delta}$-invariant function in $\mathcal{P} \mathcal{W}\left(\mathfrak{a}_{L_{i}}\right)_{N}$ which vanishes on $\left(\mathfrak{a}_{L_{i}, \mathbb{C}}^{w_{\delta, 1}}\right)^{*}$.

Proposition 4. Suppose $n$ is even, Conjecture 1 holds, and $\left\{F_{i}^{1}\right\}$ is compatible with $\left\{F_{i}^{2}\right\}$. Then there exists a function $f \in C_{c}^{\infty}(G, K)_{N}$ such that

$$
\operatorname{tr}\left(\operatorname{ind}_{P_{L_{i}}}^{G}\left(\delta \otimes e^{\nu}\right)(f) T_{k}^{\sigma_{0}}(\delta, \nu)\right)=F_{i}^{k}(\delta, \nu)
$$

for all $\delta \in\left(\hat{L}_{i}\right)_{\mathrm{lds}}, \nu \in\left(\mathfrak{a}_{L_{i}, \mathbb{C}}^{w_{\delta, k}}\right)^{*}, 1 \leqslant i \leqslant s$, and $k=1,2$.

Sketch of proof. The proof proceeds inductively as in [CD84, § 2.3]. The only obstacle is to find, for a given $\delta \in\left(\hat{L}_{j}\right)_{\mathrm{lds}}$, a function $h \in C_{c}^{\infty}(G, K)_{N}$ such that

$$
\operatorname{tr}\left(\operatorname{ind}_{P_{L_{j}}}^{G}\left(\delta_{0} \otimes e^{\nu}\right)(h) T_{k}^{\sigma_{0}}(\delta, \nu)\right)=F_{j}^{k}(\delta, \nu), \quad \nu \in\left(\mathfrak{a}_{L_{j}, \mathbb{C}}^{w_{\delta, k}}\right)^{*}, k=1,2 .
$$

We can obtain $h$ in the following manner. According to [CD90, Proposition 1], for each minimal $K$ type $\mu$ of $\operatorname{ind}_{P_{L_{j}}}^{G}\left(\delta \otimes e^{0}\right)$ there exists a function $f_{k, \mu} \in C_{c}^{\infty}(G, K)_{N}$ such that

$$
\operatorname{tr}\left(\operatorname{ind}_{P_{L_{j}}}^{G}\left(\delta \otimes e^{\nu}\right)\left(\sum_{\mu} f_{k, \mu}\right) T_{k}^{\sigma_{0}}(\delta, \nu)\right)=F_{j}^{k}(\delta, \nu), \quad \nu \in\left(\mathfrak{a}_{L_{j}, \mathbb{C}}^{w_{\delta, k}}\right)^{*} .
$$

The compatibility of $\left\{F_{i}^{1}\right\}$ with $\left\{F_{i}^{2}\right\}$ then implies that

$$
\phi_{\mu}(\nu)=\operatorname{tr}\left(\operatorname{ind}_{P_{L_{j}}}^{G}\left(\delta \otimes e^{\nu}\right)\left(f_{2, \mu}\right)\right)-\operatorname{tr}\left(\operatorname{ind}_{P_{L_{j}}}^{G}\left(\delta \otimes e^{\nu}\right)\left(f_{1, \mu}\right)\right), \quad \nu \in\left(\mathfrak{a}_{L_{j}, \mathbb{C}}^{w_{\delta_{0}, 2}}\right)^{*}
$$

vanishes on $\left(\mathfrak{a}_{L_{i}, \mathbb{C}}^{w_{\delta_{0}, 1}}\right)^{*} \cap\left(\mathfrak{a}_{L_{i}, \mathbb{C}}^{w_{\delta_{0}, 2}}\right)^{*}$. We may therefore apply Conjecture 1 to extend $\phi_{\mu}$ to a $W_{\delta}$-invariant function in $\mathcal{P W}\left(\mathfrak{a}_{L_{j}}\right)_{N}$ which vanishes on $\left(\mathfrak{a}_{L_{i}, \mathbb{C}}^{w_{\delta_{0}, 1}}\right)^{*}$. By [CD84, Proposition 1] and [Cow86], there exists a function $h_{\mu} \in C_{c}^{\infty}(G, K)_{N}$ of type $(\mu, \mu)$ such that

$$
\operatorname{tr}\left(\operatorname{ind}_{P_{L_{j}}}^{G}\left(\delta \otimes e^{\nu}\right)\left(h_{\mu}\right)\right)=\phi_{\mu}(\nu), \quad \nu \in\left(\mathfrak{a}_{L_{i}, \mathbb{C}}^{w_{\delta_{0}, 2}}\right)^{*} .
$$

Equation (11) is satisfied for $h=\sum_{\mu} h_{\mu}+f_{1, \mu}$.

\section{Applications}

Suppose $y \in G$. Obviously, $\sigma_{y} \sigma_{0} \sigma_{y}^{-1}$ is an involution of $G$ so we should have a $\sigma_{y} \sigma \sigma_{y}^{-1}$-twisted trace Paley-Wiener theorem. It is easily verified that for $\delta \in\left(\hat{L}_{i}\right)_{\mathrm{lds}}$ and $\nu \in \mathfrak{a}_{L_{i}, \mathbb{C}}^{*}$ the representation $\operatorname{ind}_{P_{L_{i}}}^{G}\left(\delta \otimes e^{\nu}\right)$ is $\sigma_{y} \sigma_{0} \sigma_{y}^{-1}$-stable if and only if it is $\sigma_{0}$-stable. If this is the case then

$$
T^{\sigma_{y} \sigma_{0} \sigma_{y}^{-1}}(\delta, \nu)=\operatorname{ind}_{P_{L_{i}}}^{G}\left(\delta \otimes e^{\nu}\right)\left(y^{-1}\right) \cdot T^{\sigma_{0}}(\delta, \nu) \cdot \operatorname{ind}_{P_{L_{i}}}^{G}\left(\delta \otimes e^{\nu}\right)(y)
$$

\footnotetext{
${ }^{1}$ We have proven a version of this conjecture in which the support of the extension is weakened to $N+\epsilon, \epsilon>0$.
} 


\section{P. MEZO}

intertwines $\operatorname{ind}_{P_{L_{i}}}^{G}\left(\delta \otimes e^{\nu}\right)$ with its $\sigma_{y} \sigma_{0} \sigma_{y}^{-1}$-conjugate. Furthermore, since taking the trace is invariant under conjugation, we have

$$
\operatorname{tr}\left(\operatorname{ind}_{P_{L_{i}}}^{G}\left(\delta \otimes e^{\nu}\right)(f) T^{\sigma}(\delta, \nu)\right)=\operatorname{tr}\left(\operatorname{ind}_{P_{L_{i}}}^{G}\left(\delta \otimes e^{\nu}\right)\left(f^{y^{-1}}\right) T^{\sigma_{y} \sigma \sigma_{y}^{-1}}(\delta, \nu)\right),
$$

where

$$
f^{y}(x)=f\left(y^{-1} x y\right), \quad x \in G,
$$

and $f \in C_{c}^{\infty}(G, K)$. This equation shows that a $\sigma_{y} \sigma \sigma_{y}^{-1}$-twisted trace Paley-Wiener theorem can be derived from a $\sigma_{0}$-twisted trace Paley-Wiener theorem of $\S \S 7$ and 8 simply by replacing $C_{c}^{\infty}(G, K)_{N}$ with $C_{c}^{\infty}\left(G, y K y^{-1}\right)_{N}$.

Let us now consider $\sigma_{0}$ as an involution of $\operatorname{GL}(n, \mathbb{R})$. As the differential of $\sigma_{0}$ sends $\lambda$ to $-\lambda$ and $g \lambda=\lambda$ for all $g \in \mathrm{GL}(n, \mathbb{R})$ and $\lambda \in \mathfrak{a}_{G}^{*}$, Langlands' classification tells us that the $\sigma_{0}$-stable representations of $\operatorname{GL}(n, \mathbb{R})$ are the $\sigma_{0}$-stable representations of $\mathrm{SL}^{ \pm}(n, \mathbb{R})$ twisted by the trivial character of $\mathfrak{a}_{G}^{*}$. We therefore obtain a $\sigma_{0}$-twisted trace Paley-Wiener theorem for $\mathrm{GL}(n, \mathbb{R})$ if we have one for $\mathrm{SL}^{ \pm}(n, \mathbb{R})$. With regard to the latter case, it is well-known that the $\mathrm{R}$ group of any discrete series representation of a Levi subgroup of $\mathrm{SL}^{ \pm}(n, \mathbb{R})$ is trivial. Consequently, if we adjust the arguments of $\S \S 4-7$ by merely ignoring any reference to $\mathrm{R}$ groups, we obtain the unique $\sigma_{0}$-twisted trace Paley-Wiener theorem for $\mathrm{SL}^{ \pm}(n, \mathbb{R})$.

To summarize, we now have $\sigma_{y} \sigma_{0} \sigma_{y}^{-1}$-twisted trace Paley-Wiener theorems on $\operatorname{SL}(n, \mathbb{R})$, $\mathrm{SL}^{ \pm}(n, \mathbb{R})$, and $\mathrm{GL}(n, \mathbb{R})$. Our remaining applications are twisted versions of the application given in $[C D 90, \S 5.1]$ in the cases that the real reductive group $\mathbf{G}(\mathbb{R})$ given there is equal to one of $\mathrm{SL}(n, \mathbb{R}), \mathrm{SL}^{ \pm}(n, \mathbb{R})$ or $\mathrm{GL}(n, \mathbb{R})$. These applications are important for the Arthur-Selberg trace formula [Art88, Proposition 1.1]. We shall give a thorough treatment of these applications only for the $\sigma_{0}$-twisted case of $\mathrm{SL}(n, \mathbb{R})$. The cases in which the underlying group is $\mathrm{SL}^{ \pm}(n, \mathbb{R})$ or $\mathrm{GL}(n, \mathbb{R})$ are simpler than the $\sigma_{0}$-twisted case of $\operatorname{SL}(n, \mathbb{R})$ and do not depend on Conjecture 1 . Conjecture 1 is also unnecessary in the $\sigma_{0}$-twisted case of $\mathrm{SL}(n, \mathbb{R})$ if $n$ is odd.

Suppose that $P=M U_{M}$ is a standard parabolic subgroup of $G$. Its normalizer $\tilde{P}$ in $G \rtimes\left\langle\sigma_{0}\right\rangle$ is readily computed to be $P$ if the block sizes $n_{1}, \ldots, n_{\ell}$ of $M$ do not satisfy

$$
n_{j}=n_{\ell+1-j}, \quad 1 \leqslant j \leqslant \ell .
$$

If the block sizes do satisfy (12) then $\tilde{P}$ is the disjoint union of $P$ and $w_{0} P \rtimes \sigma_{0}$. Similarly the normalizer $\tilde{M}$ of $M$ in $G \rtimes\left\langle\sigma_{0}\right\rangle$ is $M$, if (12) does not hold, and the disjoint union of $M$ with $w_{0} M \rtimes \sigma_{0}$ otherwise. In [Art89, $\left.\S 1\right]$, Arthur defines a Levi subset of $G \rtimes \sigma_{0}$ to be the intersection of $G \rtimes \sigma_{0}$ with $\tilde{M} \cap \tilde{P}$. In consequence, the Levi subsets of $G \rtimes \sigma_{0}$ have the form $w_{0} M \rtimes \sigma_{0}$, where $M \supset M_{0}$ is a Levi subgroup of $G$ whose block sizes satisfy (12).

Suppose $M$ satisfies (12). Then there is an obvious group isomorphism,

$$
\tilde{M}=M \cup\left(w_{0} M \rtimes \sigma_{0}\right) \cong M \rtimes\left\langle\sigma_{w_{0}} \sigma_{0}\right\rangle .
$$

We shall identify $\tilde{M}$ with the semidirect product on the right. Arthur defines $\mathfrak{a}_{M \rtimes \sigma_{w_{0}} \sigma_{0}}$ as $\operatorname{Hom}(X(M), \mathbb{R})$, where $X(M)$ is the group of rational characters of $\tilde{M}$. We compute $\mathfrak{a}_{M \rtimes \sigma_{w_{0}} \sigma_{0}}$ to be isomorphic to be the subspace

$$
\left\{X \in \mathfrak{a}_{M}: \operatorname{Ad}\left(w_{0}\right)(X)=-X\right\}=\mathfrak{a}_{M}^{w_{0}} .
$$

Given a representation $\tau$ of $\tilde{M}$ and $\lambda \in\left(\mathfrak{a}_{M, \mathbb{C}}^{w_{0}}\right)^{*}$ define $\tau_{\lambda}$ by

$$
\tau_{\lambda}(x)=\tau(x) e^{\lambda(\log (a))}, \quad x \in \tilde{M},
$$

where $a$ is the projection of $x$ onto $A_{M}$.

Let $\Pi_{\text {temp }}\left(G \rtimes \sigma_{0}\right)$ be the set of (equivalence classes of) irreducible tempered representations $\pi$ of $G \rtimes\left\langle\sigma_{0}\right\rangle$ such that the restriction of $\pi$ to $G$ is irreducible. Given a complex-valued function $\phi$ on $\Pi_{\text {temp }}\left(G \rtimes \sigma_{0}\right)$, we denote its extension to the free $\mathbb{Z}$-module generated by $\Pi_{\text {temp }}\left(G \rtimes \sigma_{0}\right)$ as $\tilde{\phi}$. 


\section{Twisted trace Paley-Wiener theorems}

Suppose $\Gamma$ is a finite set of (equivalence classes of) irreducible representations of $K$ and $N$ is a positive real number. In keeping with $[\operatorname{Art89}, \S 11]$, we define $\mathcal{I}_{N}\left(G \rtimes \sigma_{0}\right)_{\Gamma}$ to be the space of complex-valued functions $\phi$ on $\Pi_{\text {temp }}\left(G \rtimes \sigma_{0}\right)$ which satisfy the following three properties.

1) Suppose sgn is the non-trivial character of $\left\langle\sigma_{0}\right\rangle$. Then

$$
\phi(\pi \otimes \operatorname{sgn})=-\phi(\pi), \quad \pi \in \Pi_{\text {temp }}\left(G \rtimes \sigma_{0}\right) .
$$

2) Suppose that the restriction of $\pi \in \Pi_{\text {temp }}\left(G \rtimes \sigma_{0}\right)$ to $K$ does not contain any representation of $\Gamma$. Then $\phi(\pi)=0$.

3) Suppose that $w_{0} M \rtimes \sigma_{0}$ is a Levi subset $G \rtimes \sigma_{0}$ and that $\tau$ is an irreducible tempered representation of $\tilde{M}$ which remains irreducible when restricted to $M$. Then the integral

$$
\phi(\tau, X)=\int_{i\left(\mathfrak{a}_{M}^{w_{0}}\right)^{*}} \tilde{\phi}\left(\operatorname{ind}_{\tilde{P}}^{G \rtimes\left\langle\sigma_{0}\right\rangle}\left(\tau_{\lambda}\right)\right) e^{-\lambda(X)} d \lambda, \quad X \in \mathfrak{a}_{M}^{w_{0}}
$$

converges to a smooth function of $X$ which has support in the closed ball of radius $N$ centered about the origin in $\mathfrak{a}_{M}^{w_{0}}$.

Define $C_{c}^{\infty}(G, K)_{N, \Gamma}$ to be the subspace of functions of $C_{c}^{\infty}(G, K)_{N}$ which transform according to representations occurring in $\Gamma$ under the bilateral action of $K$.

Theorem 2. Suppose Conjecture 1 is true if $n$ is even. Suppose $f \in C_{c}^{\infty}(G, K)_{N, \Gamma}$ and

$$
\phi(f)(\pi)=\operatorname{tr}\left(\int_{G} f(x) \pi\left(x, \sigma_{0}\right) d x\right), \quad \pi \in \Pi_{\text {temp }}\left(G \rtimes \sigma_{0}\right) .
$$

Then the map given by $f \mapsto \phi(f)$ is surjective onto $\mathcal{I}_{N}\left(G \rtimes \sigma_{0}\right)_{\Gamma}$.

Proof. Using the notation of $\S 7$, suppose that $1 \leqslant i \leqslant s, 0 \leqslant t \leqslant\lfloor n / 2\rfloor, L_{i} \in \mathcal{L}(t), \delta \in\left(\hat{L}_{i}\right)_{\operatorname{lds}, t}^{1}, w_{t}$ is the longest element in $Z_{W_{\delta}}\left(R_{\delta}\right)$, and $\nu \in i\left(\mathfrak{a}_{L_{i}}^{w t}\right)^{*}$. Then $\operatorname{ind}_{P_{L_{i}}}^{G}\left(\delta \otimes e^{v}\right)$ is $\sigma_{0}$-stable and decomposes as a finite sum,

$$
\operatorname{ind}_{P_{L_{i}}}^{G}\left(\delta \otimes e^{v}\right)=\pi_{1}^{0} \oplus \cdots \oplus \pi_{m}^{0},
$$

of irreducible tempered representations of $G$. The corresponding intertwining operator, $T_{1}^{\sigma_{0}}(\delta, \nu)$, can be used to define representations $\pi_{1}, \ldots, \pi_{m} \in \Pi_{\text {temp }}\left(G \rtimes \sigma_{0}\right)$ by setting $T_{j}$ equal to the restriction of $T_{1}^{\sigma_{0}}(\delta, \nu)$ to the space of $\pi_{j}^{0}$ and

$$
\pi_{j}\left(x, \sigma_{0}\right)=\pi_{j}^{0}(x) T_{j}, \quad x \in G .
$$

We define

$$
F_{i, t}^{1}(\delta, \nu)=\phi\left(\pi_{1}\right)+\cdots+\phi\left(\pi_{m}\right) .
$$

This procedure can be repeated with $W_{\delta}^{0}$ in place of $Z_{W_{\delta}}\left(R_{\delta}\right)$, and $T_{2}(\delta, \nu)$ in place of $T_{1}(\delta, \nu)$ to define

$$
F_{i, t}^{2}(\delta, \nu)=\phi\left(\pi_{1}\right)+\cdots+\phi\left(\pi_{m}\right), \quad \delta \in\left(\hat{L}_{i}\right)_{\mathrm{lds}, t}^{2}, \quad \nu \in i\left(\mathfrak{a}_{L_{i}}^{w_{t}}\right)^{*}
$$

(we apologize for the use of $i$ as an index as well as the customary imaginary number). Recall the Levi subgroup $M_{L_{i}, t}$ defined in $\S 4$. By construction, the block sizes of $M_{L_{i}, t}$ satisfy (12) and $\mathfrak{a}_{M_{L_{i}, t}}^{w_{0}}=\mathfrak{a}_{L_{i}}^{w_{t}}$. In addition, the representations

$$
\operatorname{ind}_{P_{L_{i}} \cap M_{L_{i}, t}}^{M_{L_{i}, t}}\left(\delta \otimes e^{\nu}\right)=\left(\operatorname{ind}_{P_{L_{i}} \cap M_{L_{i}, t}^{1}}^{M_{L_{i}, t}^{1}} \delta\right) \otimes e^{\nu}, \quad \delta \in\left(\hat{L}_{i}\right)_{\text {lds }, t}^{k}, \quad \nu \in i\left(\mathfrak{a}_{L_{i}}^{w_{t}}\right)^{*}, \quad k=1,2,
$$

are irreducible, as their $\mathrm{R}$ groups are trivial. Using the ideas of $\S 4$, it is simple to show that these operators are $\sigma_{w_{0}} \sigma_{0}$-stable. Therefore, for every $\nu \in i\left(\mathfrak{a}_{L_{i}}^{w_{t}}\right)^{*}$ we may define a representation $\tau_{\nu}$ of 
the Levi subset $\tilde{M}=\tilde{M}_{L_{i}, t}$ such that its restriction to $M_{L_{i}, t}$ is equal to $\operatorname{ind}_{P_{L_{i}} \cap M_{L_{i}, t}^{1}}^{M_{L_{i}}^{1}}\left(\delta \otimes e^{\nu}\right)$ and

$$
\tilde{\phi}\left(\operatorname{ind}_{\tilde{P}}^{G \rtimes \sigma_{0}}\left(\tau_{\nu}\right)\right)=F_{i, t}^{k}(\delta, \nu), \quad k=1,2 .
$$

Property 3 of $\phi$ implies that $F_{i, t}^{1}(\delta, \cdot)$ and $F_{i, t}^{2}(\delta, \cdot)$ extend to functions of complex variables in $\mathcal{P W}\left(\mathfrak{a}_{L_{i}}^{w_{t}}\right)_{N}$.

Retaining the same notation for these extensions, we now have functions

$$
F_{i, t}^{k}:\left(\hat{L}_{i}\right)_{\mathrm{lds}, t}^{k} \times\left(\mathfrak{a}_{L_{i}, \mathbb{C}}^{w_{t}}\right)^{*} \rightarrow \mathbb{C}, \quad k=1,2
$$

for all $1 \leqslant i \leqslant s$ such that $L_{i} \in \mathcal{L}(t)$, which satisfy condition $\mathrm{b}(2)$ of Theorem 1 . We wish to show that these functions satisfy the other conditions of Theorem 1(b). In the proof of Theorem 1 we appeal to the result of Harish-Chandra which tells us that there are only finitely many inequivalent irreducible admissible representations of $L_{i}$ containing a fixed $\left(K \cap L_{i}\right)$-type. This result and property 2 of $\phi$ imply that our functions satisfy the finite support condition of Theorem $1 b(1)$. The remaining two conditions, Theorem $1 \mathrm{~b}(3)$ and (4), are easily seen to be satisfied from the definitions of $T_{k}^{\sigma_{0}}(\delta, \nu)$, as these families of operators have been defined to be compatible under conjugation by permutation matrices (cf. Theorem $1 \mathrm{~b}(3)$ ), and compatible under affiliation (cf. Theorem $1 \mathrm{~b}(4)$ ).

As we know from $\S 5$, for any $k=1,2$ and $1 \leqslant j \leqslant s$, every $\delta \in\left(\hat{L}_{j}\right)_{\text {lds }}$ is conjugate to a representation in $\left(\hat{L}_{i}\right)_{\text {lds }, t}^{k}$, for some $L_{i} \in \mathcal{L}(t)$. We can therefore define functions

$$
F_{j}^{k}:\left(\hat{L}_{j}\right)_{\mathrm{lds}} \times\left(\mathfrak{a}_{L_{j}, \mathbb{C}}^{w_{\delta, k}}\right)^{*} \rightarrow \mathbb{C}, \quad 1 \leqslant j \leqslant s, k=1,2,
$$

satisfying the conditions of Corollary $1(\mathrm{~b})$, from the functions of $\left\{F_{i, t}^{k}\right\}$ in an obvious manner. By Proposition 4, the theorem is proven if we show that $\left\{F_{j}^{1}\right\}$ is compatible with $\left\{F_{j}^{2}\right\}$. Suppose, therefore, that the $\mathrm{R}$ group of $\delta \in\left(\hat{L}_{j}\right)_{\mathrm{lds}}$ is trivial. This implies that for any $\nu \in i\left(\mathfrak{a}_{L_{j}}^{w_{\delta, 1}}\right)^{*}$, the induced representation

$$
\pi^{0}=\operatorname{ind}_{P_{L_{j}}}^{G}\left(\delta \otimes e^{\nu}\right)
$$

is an irreducible tempered representation. Combined with each of the intertwining operators, $T_{1}^{\sigma_{0}}(\delta, \nu)$ and $T_{2}^{\sigma_{0}}(\delta, \nu)$, the representation $\pi^{0}$ determines respective representations $\pi_{1}$ and $\pi_{2}$ in $\Pi_{\text {temp }}\left(G \rtimes \sigma_{0}\right)$. By definition, $F_{j}^{k}(\delta, \nu)=\phi\left(\pi_{k}\right)$ for $k=1,2$. If $T_{1}^{\sigma_{0}}(\delta, \nu)=T_{2}^{\sigma_{0}}(\delta, \nu)$ then, by definition (cf. $\S 7$ ), $c_{\delta}=1$ and

$$
F_{j}^{2}(\delta, \nu)=\phi\left(\pi_{2}\right)=\phi\left(\pi_{1}\right)=c_{\delta} F_{j}^{1}(\delta, \nu) .
$$

Otherwise, $\pi_{2}=\pi_{1} \otimes \operatorname{sgn}, c_{\delta}=-1$, and, by property 1 of $\phi$, we have

$$
F_{j}^{2}(\delta, \nu)=\phi\left(\pi_{2}\right)=\phi\left(\pi_{1} \otimes \operatorname{sgn}\right)=-\phi\left(\pi_{1}\right)=c_{\delta} F^{1}(\delta, \nu) .
$$

Hence, $\left\{F_{j}^{1}\right\}$ is compatible with $\left\{F_{j}^{2}\right\}$ and the theorem is complete.

\section{ACKNOWLEDGEMENTS}

The author would like to thank the Weizmann Institute of Science and the University of Toronto for their generous support, and S. Gelbart and J. Repka for their encouragement.

\section{REFERENCES}

Art88 J. Arthur, The invariant trace formula I. Local theory, J. Amer. Math. Soc. 1 (1988), 323-383.

Art89 J. Arthur, Intertwining operators and residues I, J. Funct. Anal. 84 (1989), 19-84.

CD84 L. Clozel and P. Delorme, Le théorème de Paley-Wiener invariant pour les groupes de Lie réducifs, Invent. Math. 77 (1984), 427-453. 


\section{Twisted trace Paley-Wiener theorems}

CD90 L. Clozel and P. Delorme, Le théorème de Paley-Wiener invariant pour les groupes de Lie réducifs II, Ann. Sci. École Norm. Sup (4) 23 (1990), 193-228.

Cow86 M. Cowling, On the Paley-Wiener theorem, Invent. Math. 83 (1986), 403-404.

Del91 P. Delorme, Théorème de Paley-Wiener invariant tordu pour le changement de base $\mathbb{C} / \mathbb{R}$, Compositio Math. 80 (1991), 197-228.

Hum72 J. E. Humphreys, Introduction to Lie algebras and representation theory (Springer-Verlag, 1972).

Kna79 A. Knapp, Representations of $\mathrm{GL}_{2}(\mathbb{R})$ and $\mathrm{GL}_{2}(\mathbb{C})$, in Automorphic forms, representations, and L-functions, Proc. Sympos. Pure Math. XXXIII (1979), 87-91.

Kna86 A. Knapp, Representation theory of semisimple groups (Princeton University Press, 1986).

Lan89 R. P. Langlands, On the classification of irreducible representations of real algebraic groups, in Representation theory and harmonic analysis on semisimple Lie groups, Math. Surveys Monogr. vol. 31 (American Mathematical Society, 1989), 101-170.

SV80 B. Speh and D. A. Vogan, Reducibility of generalized principal series representations, Acta Math. 145 (1980), 227-299.

Paul Mezo pmezo@math.toronto.edu

Fields Institute, 222 College Street, Toronto, Ontario, M5T 3J1, Canada 